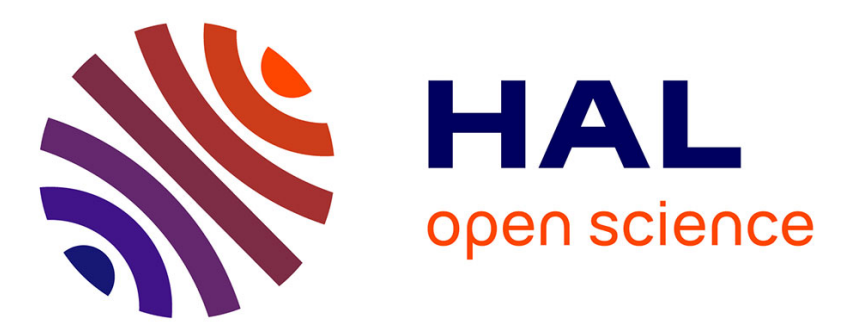

\title{
Approximate local magnetic-to-electric surface operators for time-harmonic Maxwell's equations
}

\author{
Mohamed El Bouajaji, Xavier Antoine, Christophe Geuzaine
}

\section{To cite this version:}

Mohamed El Bouajaji, Xavier Antoine, Christophe Geuzaine. Approximate local magnetic-to-electric surface operators for time-harmonic Maxwell's equations. Journal of Computational Physics, 2014, 279 (15), pp.241-260. 10.1016/j.jcp.2014.09.011 . hal-01094824

\section{HAL Id: hal-01094824 \\ https://hal.science/hal-01094824}

Submitted on 9 Feb 2016

HAL is a multi-disciplinary open access archive for the deposit and dissemination of scientific research documents, whether they are published or not. The documents may come from teaching and research institutions in France or abroad, or from public or private research centers.
L'archive ouverte pluridisciplinaire $\mathbf{H A L}$, est destinée au dépôt et à la diffusion de documents scientifiques de niveau recherche, publiés ou non, émanant des établissements d'enseignement et de recherche français ou étrangers, des laboratoires publics ou privés. 


\title{
Approximate local magnetic-to-electric surface operators for time-harmonic Maxwell's equations
}

\author{
M. El Bouajaji *† X. Antoine ${ }^{* \dagger}$, C. Geuzaine ${ }^{\ddagger}$
}

\begin{abstract}
The aim of this paper is to propose new local and accurate approximate magnetic-to-electric surface boundary operators for the three-dimensional time-harmonic Maxwell's equations. After their construction where their accuracy is improved through a regularization process, a localization of these operators and a full finite element approximation is introduced. Next, their numerical efficiency and accuracy is investigated in detail for different scatterers when these operators are used in the extreme situation of On-Surface Radiation Conditions methods.
\end{abstract}

\section{Contents}

\begin{tabular}{|lll}
\hline 1 & Introduction & 1 \\
\hline
\end{tabular}

2 Problem setting and integral representations

3 Approximate MtE surface operators for a general smooth surface $\Gamma \quad 4$

3.1 Surface operators and Sobolev spaces . . . . . . . . . . . . . . . . . . . 4

3.2 Construction of nonlocal approximate MtE operators: from the half-plane case to a general surface . . . . . . . . . . . . . . . . . . . . . . 5

3.3 Construction of nonlocal approximate MtE operators: accuracy improvement by regularization.

4 Localization and discretization of the MtE operators

4.1 Local representation of the MtE operators . . . . . . . . . . . . . . . . . . . . . 11

4.2 Finite element discretization . . . . . . . . . . . . . . . . . . . . . . . . . . 14

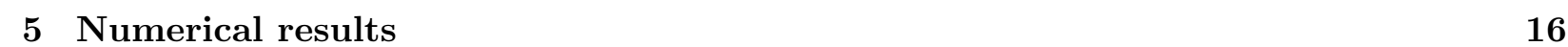

\begin{tabular}{|rr}
\hline 6 & Conclusion \\
\hline
\end{tabular}

\section{Introduction}

Time harmonic electromagnetic scattering is an important area of research because of its numerous scientific and technological applications. In terms of computational methods, solving threedimensional electromagnetic scattering problems is known to be a challenging topic, most particularly in the high frequency regime. Various approaches can be used to numerically obtain the

\footnotetext{
${ }^{*}$ Université de Lorraine, Institut Elie Cartan de Lorraine, UMR 7502, Vandoeuvre-lès-Nancy, F-54506, France. (mohamed.el_bouajaji@inria.fr, xavier.antoine@univ-lorraine.fr).

${ }^{\dagger}$ Inria Nancy Grand-Est/IECL - ALICE.

${ }^{\ddagger}$ Université de Liège, Institut Montefiore B28, Grande Traverse 10, B-4000 Liège, Belgium. (cgeuzaine@ulg.ac.be)
} 
electromagnetic fields diffracted by an object. Among the most widely used methods, let us mention e.g. the finite element method [36, 24] with an Absorbing Boundary Condition (ABC) [36] or a Bérenger's Perfectly Matched Layer (PML) [36, 18, 25] or the integral equation formulations [48, 40, 23] discretized by boundary element methods, in conjunction with the Multilevel Fast Multipole Method (MFMM) [24, 40, 29, 28] and Krylov subspace solvers [54]. Of course, other approaches may be considered like for example asymptotic methods [4].

In all case, the success of a numerical method for prospecting high frequency scattering is based on the fact that the method is stable and well conditioned [23] with respect to the wave number $k$. Different strategies can be adopted for the stabilization of a numerical method. For example, for preconditioning, many developments have been made over the last two decades from the algebraic point of view [54]. However, when $k$ is large, these techniques are limited in terms of stability because the mathematical structure and physics of the underlying operator, that is the Maxwell operators, are lost during the purely algebraic operations. To overcome this problem, recent promising directions [11, 12, 13, 10, 9, 21, 22, 2, 19, 39] have emerged by producing new preconditioning techniques based on (pseudo differential) operators calculus and integral equations that are then discretized to get a matrix representation. One crucial point in these approaches is to build approximate and accurate representations of the operators linking the magnetic (M) and the electric $(\mathbf{J})$ surface currents through the so-called Magnetic-to-Electric (MtE) map [48]: $\operatorname{MtE}(\mathbf{M}, \mathbf{J})=\mathbf{0}$ on $\Gamma$, where $\Gamma$ is the scattering surface. It is quite natural that this map plays a key role in a numerical method since the scattered electromagnetic fields can be computed a posteriori through the Stratton-Chu formulae (3) once these the quantities $\mathbf{M}$ and $\mathbf{J}$ are determined. When an approximation of the MtE surface operator is known, it can be suitably injected into a standard integral equation for preconditioning or can be used to build new well-conditioned and stable integral equations with respect to $k$. These directions have now been widely studied in the case of three-dimensional acoustics [10, 9, 11, 12, 13, 21, 22, 2, 19, 39], where the MtE operator is then the Dirichlet-to-Neumann map $\left(\operatorname{DtN}\left(u, \partial_{\mathbf{n}} u\right)=0\right.$ on $\left.\Gamma\right)$ [56, 35, 34, 14, 7], but much less deeply for electromagnetic scattering [27, 21, 22, 2, 19, 51. Other applications of approximate DtN operators in acoustics include ABCs [7, 37, 52, 16, 15, 44, 57, 17, 56, 35, 34 techniques for domain truncation and Domain Decomposition Methods (DDM) [33, 30, 31, 42, 41, 50, 49, 20]. In these two last situations, the DtN/MtE surface operators are applied on a fictitious boundary $\Sigma$ and not $\Gamma$. A last potential application is related to the construction of generalized impedance boundary operators for scattering by dielectric obstacles [55, 8].

The goal of the present paper is to propose some accurate and local approximations of MtE surface operators for the three-dimensional time harmonic Maxwell's equation. In section 2, we set the scattering problem and explain why MtE maps are important in a practical calculation, most particularly because of the central role played by the Stratton-Chu integral equations representations. Section 3 is devoted to the construction of approximate nonlocal MtE surface operators on a general shape $\Gamma$. We first begin in subsection 3.1 by introducing the standard surface operators that will be needed in the sequel of the paper. Next, in subsection 3.2, we develop the full analysis for building the exact MtE maps for the half-space case through Fourier analysis. We also discuss the extension to a general shape $\Gamma$. In this latter case, the two operators that we propose are no longer exact but approximate. To increase their accuracy, we introduce in subsection 3.3 a regularization process of the local and nonlocal operators that define the MtE maps. Numerical simulations based on the calculation of the exact electromagnetic fields for a sphere show that an optimal regularization parameter must be carefully chosen. We obtain a precise estimate of this parameter through an asymptotic analysis of the reflection coefficients associated with the exact solution. Section 4 discusses the numerical approximation of these MtE operators by first using a localization process based on complex rational Padé approximants (section 4.1) and next finite element discretizations (section 4.2). Section 5 provides various numerical simulations of these new MtE surface operators when they are used in the extreme context of On-Surface Radiation Condition (OSRCs) methods 
[38, 6, 44, 17, 52, 5, 14. Let us mention that only a few results are available in the literature [27, 47, 3, 53] when the OSRC technique is used in the framework of the full set of Maxwell's equations. Indeed, these papers only derive special canonical solutions but not a general and flexible approach as the one presented here for a general surface $\Gamma$. We mainly discuss the efficiency and accuracy of the methods when the local MtE operators are used. This allows us to show that fast and accurate OSRCs solutions (surface fields and far-field patterns computations) can be obtained and that these operators should be useful when applied in the context of preconditioning, DDM and other hybrid computational techniques for electromagnetic scattering.

\section{Problem setting and integral representations}

Let $\Omega_{i}$ be a bounded scatterer in $\mathbb{R}^{3}$ with a smooth closed boundary $\Gamma$. The associated unbounded domain of propagation is denoted by $\Omega_{e}:=\mathbb{R}^{3} \backslash \overline{\Omega_{i}}$. The exterior electromagnetic scattering problem by a Perfect Electrically Conducting (PEC) body $\Omega_{i}$ writes down

$$
\left\{\begin{array}{l}
\operatorname{curl} \mathbf{H}+\imath k Z_{0}^{-1} \mathbf{E}=0, \text { in } \Omega_{e}, \\
\operatorname{curl} \mathbf{E}-\imath k Z_{0} \mathbf{H}=0, \text { in } \Omega_{e}, \\
\mathbf{n} \times(\mathbf{n} \times \mathbf{E})=-\mathbf{n} \times\left(\mathbf{n} \times \mathbf{E}^{\text {inc }}\right), \text { on } \Gamma, \\
\lim _{r \rightarrow \infty} r\left(\mathbf{E}+Z_{0} \hat{\mathbf{x}} \times \mathbf{H}\right)=0 .
\end{array}\right.
$$

In the above equations, $\mathbf{H}:=\left(H_{1}, H_{2}, H_{3}\right)^{t}$ and $\mathbf{E}:=\left(E_{1}, E_{2}, E_{3}\right)^{t}$ denote the scattered magnetic and electric fields, respectively. The wavenumber is $k:=2 \pi / \lambda$, where $\lambda$ is the wavelength. The real valued parameter $Z_{0}$ is the impedance of the exterior media $\Omega_{e}$ and the unit imaginary number is $\imath=\sqrt{-1}$. Vector $\mathbf{n}$ is the unit outwardly directed normal to $\Omega_{i}$. The notation $\mathbf{a} \times \mathbf{b}$ designates the cross product and $\mathbf{a} \cdot \overline{\mathbf{b}}$ the inner product between two complex valued vector fields $\mathbf{a}$ and $\mathbf{b}$ in $\mathbb{C}^{3}$, where $\bar{z}$ is the complex conjugate of $z \in \mathbb{C}$. The associated norm is $\|\mathbf{a}\|:=\sqrt{\mathbf{a} \cdot \overline{\mathbf{a}}}$. Vector $\mathbf{E}^{\text {inc }}$ defines a given incident electric field. The rotational operator is denoted by curl. Let us now define $\mathbf{x}=\left(x_{1}, x_{2}, x_{3}\right)^{t}=r \hat{\mathbf{x}} \in \mathbb{R}^{3}$, with $r:=\|\mathbf{x}\|$ and $\hat{\mathbf{x}}$ the directional vector of the unit sphere $\mathbb{S}_{1}$. Then, the last equation of system (1), the so-called Silver-Müller radiation condition at infinity, provides the uniqueness of the solution to the boundary-value problem. More precisely, it can be proved [46, 48] that the solution $(\mathbf{E}, \mathbf{H})$ to the boundary-value problem (1) is unique in the functional space $\mathbf{H}_{\mathrm{loc}}\left(\operatorname{curl}, \Omega_{e}\right) \times \mathbf{H}_{\mathrm{loc}}\left(\operatorname{curl}, \Omega_{e}\right)$ once $\mathbf{E}^{\text {inc }}$ is an element of $\mathbf{H}_{\mathrm{loc}}\left(\operatorname{curl}, \Omega_{e}\right):=\left\{\mathbf{v} \in \mathbf{L}_{\mathrm{loc}}^{2}\left(\Omega_{e}\right) /\right.$ $\left.\operatorname{curl} \mathbf{v} \in \mathbf{L}_{\mathrm{loc}}^{2}\left(\Omega_{e}\right)\right\}$.

The free-space Green's function $G$ is given in the three-dimensional case by

$$
G(\mathbf{x}, \mathbf{y})=\frac{\exp (\imath k\|\mathbf{x}-\mathbf{y}\|)}{4 \pi\|\mathbf{x}-\mathbf{y}\|}, \mathbf{x} \neq \mathbf{y} .
$$

Let us introduce the surface electric and magnetic currents $\mathbf{J}=\mathbf{n} \times \mathbf{E}$ and $\mathbf{M}=\mathbf{n} \times \mathbf{H}$, respectively. Then, one way to solve system (1) is based on writing well-posed integral equation formulations starting from the Stratton-Chu representation formulae [26]

$$
\begin{aligned}
& \mathbf{E}(\mathbf{x}):=\imath k Z_{0} \mathcal{T} \mathbf{M}(\mathbf{x})-\mathcal{K} \mathbf{J}(\mathbf{x}), \\
& \mathbf{H}(\mathbf{x}):=-\mathcal{K} \mathbf{M}(\mathbf{x})-\imath k Z_{0}^{-1} \mathcal{T} \mathbf{J}(\mathbf{x}),
\end{aligned}
$$

for any $\mathbf{x} \in \Omega_{e}$. The electric and magnetic potentials $\mathcal{T}$ and $\mathcal{K}$ are respectively given by

$$
\begin{aligned}
\mathcal{T} \mathbf{M}(\mathbf{x}) & :=\frac{1}{k^{2}} \operatorname{curl}_{\mathbf{x}} \operatorname{curl}_{\mathbf{x}} \int_{\Gamma} G(\mathbf{x}, \mathbf{y}) \mathbf{M}(\mathbf{y}) d \Gamma(\mathbf{y}), \\
\mathcal{K} \mathbf{J}(\mathbf{x}) & :=-\operatorname{curl}_{\mathbf{x}} \int_{\Gamma} G(\mathbf{x}, \mathbf{y}) \mathbf{J}(\mathbf{y}) d \Gamma(\mathbf{y}) .
\end{aligned}
$$


From (2)-(3)-(4), we can deduce a surface integral equation [24] to solve on $\Gamma$ (an EFIE or CFIE for instance), according to the unknowns $\mathbf{J}$ and $\mathbf{M}$. We can thus compute the scattered fields from (3). In particular, we have the following asymptotic expression in the far-field region

$$
\mathbf{E}(\mathbf{x})=\frac{\exp (\imath k r)}{r}\left(\mathbf{E}_{\infty}(\hat{\mathbf{x}})+\mathcal{O}\left(\frac{1}{r}\right)\right) \quad \text { as } r \rightarrow \infty,
$$

where the electric far-field pattern $\mathbf{E}_{\infty}$ is defined by

$$
\mathbf{E}_{\infty}(\hat{\mathbf{x}})=\frac{\imath k}{4 \pi} \int_{\Gamma}(\mathbf{J}(\mathbf{y})+\mathbf{M}(\mathbf{y}) \times \hat{\mathbf{x}}) \exp (-\imath k \hat{\mathbf{x}} \cdot \mathbf{y}) d \Gamma(\mathbf{y})
$$

As already told, the computation of $\mathbf{J}$ and $\mathbf{M}$ on $\Gamma$ is based on an integral equation. Since the operators defining such an equation are nonlocal, the computational cost required to obtain an approximation of these two quantities can be huge, most particularly for large wave numbers $k$ (high frequency regime). Of course, modern computational strategies combine a fast evaluation algorithm $(\mathrm{FMM}, \ldots[24,40,29,28])$ and a preconditioned Krylov solver [54] but this is still extremely memory and time consuming most particularly in the high frequency regime. For this reason, being able to design a representation like

$$
\mathscr{M} \mathbf{M}+\mathscr{J} \mathbf{J}=\mathbf{0}, \text { on } \Gamma,
$$

with $\mathscr{M}$ and $\mathscr{J}$ two inversible local boundary operators is clearly useful since the corresponding discrete part would correspond to sparse matrices. In fact, as we will see later (section 3), local operators are not enough but explicit non local operators that are "easy-to-localize" implicitly can be obtained (see Section 4.1). Of course, in Equation (7), the corresponding surface fields $\mathbf{M}$ and $\mathbf{J}$ are not exact but approximate, even at the continuous level, and should therefore be labeled $\widetilde{\mathbf{M}}$ and $\widetilde{\mathbf{J}}$ (we often omit the tilde when there is no ambiguity in the discussions). Having a relation like (7) makes the method fall into the class of On-Surface Radiation Conditions (OSRCs) methods [?]. An approximate relation linking $\mathbf{M}$ and $\mathbf{J}$ is called an approximate Magnetic-to-Electric (MtE) surface map.

\section{Approximate MtE surface operators for a general smooth sur- face $\Gamma$}

The construction of the non-local boundary condition $(7)$ through the maps $\mathscr{M}$ and $\mathscr{J}$ is realized in two steps. First, we consider the half-space case where Fourier analysis allows us to build some exact operators. Next, we propose the extension to a three-dimensional smooth surface $\Gamma$ by using the local tangent plane approximation of the surface (section 3.2) and a regularization procedure of a square-root operator (section 3.3). The numerical localization of the operator as well as its finite element approximation and numerical validation are treated in Section 4.

\subsection{Surface operators and Sobolev spaces}

In what follows we need some surface operators and their functional background for a general smooth three-dimensional surface $\Gamma$. Let us define the tangential gradient $\nabla_{\Gamma}$ which acts from $H^{1 / 2}(\Gamma)$ to $\mathbf{H}_{\times}^{-1 / 2}(\Gamma)$, where $\mathbf{H}_{\times}^{s}(\Gamma):=\left\{\mathbf{v} \in \mathbf{H}^{s}(\Gamma) / \mathbf{v} \cdot \mathbf{n}=0\right.$ on $\left.\Gamma\right\}=\mathbf{n} \times \mathbf{H}^{s}$, for $s \in \mathbb{R}$. The tangential vector curl operator $\mathbf{c u r l}_{\Gamma}$ acts from $H^{1 / 2}(\Gamma)$ to $\mathbf{H}_{\times}^{-1 / 2}(\Gamma)$ (the duality is defined with respect to the $L^{2}$ - and $\mathbf{L}^{2}$-inner products). These continuous operators have dual operators $\operatorname{div}_{\Gamma}$

and $\operatorname{curl}_{\Gamma}$, respectively, acting from $\mathbf{H}_{\times}^{1 / 2}(\Gamma)$ to $H^{-1 / 2}(\Gamma)$. The scalar Laplace-Beltrami operator is defined by $\Delta_{\Gamma}:=\operatorname{div}_{\Gamma} \nabla_{\Gamma}=-\operatorname{curl}_{\Gamma} \operatorname{curl}_{\Gamma}$. The vectorial Laplace-Beltrami operator satisfies $\boldsymbol{\Delta}_{\Gamma}=\nabla_{\Gamma} \operatorname{div}_{\Gamma}-\operatorname{curl}_{\Gamma} \operatorname{curl}_{\Gamma}$. Let us denote by $\mathbf{H}_{\times}^{-1 / 2}\left(\operatorname{div}_{\Gamma}, \Gamma\right):=\left\{\mathbf{v} \in \mathbf{H}_{\times}^{-1 / 2}(\Gamma) / \operatorname{div}_{\Gamma} \mathbf{v} \in H^{-1 / 2}(\Gamma)\right\}$ 
the Hilbert space of well-defined surface divergence fields and by $\mathbf{H}_{\times}^{-1 / 2}\left(\operatorname{curl}_{\Gamma}, \Gamma\right):=\left\{\mathbf{v} \in \mathbf{H}_{\times}^{-1 / 2}(\Gamma) /\right.$ $\left.\operatorname{curl}_{\Gamma} \mathbf{v} \in H^{-1 / 2}(\Gamma)\right\}$ its dual. Finally, let us consider a smooth vector field $\mathbf{v} \in \mathbf{C}^{\infty}(\bar{\Omega})$. We define the tangential traces applications by

$$
\gamma_{t}: \mathbf{v} \mapsto \mathbf{v}_{t}:=\mathbf{v}_{\mid \Gamma} \times \mathbf{n} \text { and } \gamma_{T}: \mathbf{v} \mapsto \mathbf{v}_{T}:=\mathbf{n} \times\left(\mathbf{v}_{\mid \Gamma} \times \mathbf{n}\right)=\mathbf{v}_{\mid \Gamma}-\left(\mathbf{v}_{\mid \Gamma} \cdot \mathbf{n}\right) \mathbf{n} .
$$

In the case of a smooth domain, the applications $\gamma_{t}$ and $\gamma_{T}$ can be extended by continuity to surjective linear applications from $\mathbf{H}(\mathbf{c u r l}, \Omega)$ to $\mathbf{H}_{\times}^{-1 / 2}\left(\operatorname{div}_{\Gamma}, \Gamma\right)$ and $\mathbf{H}_{\times}^{-1 / 2}\left(\operatorname{curl}_{\Gamma}, \Gamma\right)$, respectively.

\subsection{Construction of nonlocal approximate MtE operators: from the half-plane case to a general surface}

Let us consider that $\Gamma:=\left\{\mathbf{x} \in \mathbb{R}^{3} / x_{1}=0\right\}$. We denote by $\mathbf{n}:=(1,0,0)$ the outwardly directed unit normal vector at $\Gamma$ to $\Omega_{i}:=\left\{\mathbf{x} \in \mathbb{R}^{3} / x_{1}<0\right\}$. If we consider that $x_{1}$ is the radial direction to $\Omega_{i}$, then the tangential direction is $\mathbf{x}_{\|}:=\left(x_{2}, x_{3}\right)$. In the following, we define the partial Fourier transform $\hat{\mathbf{f}}$ of a function $\mathbf{f}:=\left(f_{1}, f_{2}, f_{3}\right): \mathbb{R}^{3} \rightarrow \mathbb{R}^{3}$ by

$$
\hat{f}_{j}\left(x_{1}, \boldsymbol{\xi}\right):=\int_{\mathbb{R}^{2}} f_{j}\left(x_{1}, \mathbf{x}_{\|}\right) e^{-i \mathbf{x}_{\|} \cdot \boldsymbol{\xi}} d \mathbf{x}_{\|}, \quad j=2,3
$$

and the inverse Fourier transform by

$$
f_{j}(\mathbf{x}):=\frac{1}{(2 \pi)^{2}} \int_{\mathbb{R}^{2}} \hat{f}_{j}\left(x_{1}, \boldsymbol{\xi}\right) e^{i \mathbf{x}_{\|}} \cdot \boldsymbol{\xi} d \boldsymbol{\xi}, \quad j=2,3 .
$$

The dual variable of $\mathbf{x}_{\|}$is $\boldsymbol{\xi}:=\left(\xi_{2}, \xi_{3}\right)$ and we set : $\|\boldsymbol{\xi}\|:=\sqrt{\boldsymbol{\xi} \cdot \boldsymbol{\xi}}$. Under the above notations, we can state the following proposition.

Proposition 1. For $\Gamma:=\left\{\mathbf{x} \in \mathbb{R}^{3} / x_{1}=0\right\}$, we have the following exact MtE surface relation

$$
\mathbf{M}+\boldsymbol{\Lambda}^{\mathrm{ex}}(\mathbf{n} \times \mathbf{J})=0 \text {, on } \Gamma,
$$

where the operator $\boldsymbol{\Lambda}^{\mathrm{ex}}$ is defined by

$$
\boldsymbol{\Lambda}^{\mathrm{ex}}=\frac{1}{Z_{0}}\left(\mathbf{I}+\frac{1}{k^{2}} \boldsymbol{\Delta}_{\Gamma}\right)^{-\frac{1}{2}}\left(\mathbf{I}-\frac{1}{k^{2}} \mathbf{c u r l}_{\Gamma} \operatorname{curl}_{\Gamma}\right),
$$

and $\mathbf{I}$ is the tangent plane identity operator.

Proof. We begin by developing the Maxwell's system in (1)

$$
\left\{\begin{array}{lll}
-i k Z_{0} H_{1}+\frac{\partial E_{3}}{\partial x_{2}}-\frac{\partial E_{2}}{\partial x_{3}}= & 0, & i k Z_{0}^{-1} E_{1}+\frac{\partial H_{3}}{\partial x_{2}}-\frac{\partial H_{2}}{\partial x_{3}}=0, \\
-i k Z_{0} H_{2}+\frac{\partial E_{1}}{\partial x_{3}}-\frac{\partial E_{3}}{\partial x_{1}}=0, & i k Z_{0}^{-1} E_{2}+\frac{\partial H_{1}}{\partial x_{3}}-\frac{\partial H_{3}}{\partial x_{1}}=0, \\
-i k Z_{0} H_{3}+\frac{\partial E_{2}}{\partial x_{1}}-\frac{\partial E_{1}}{\partial x_{2}}=0, & i k Z_{0}^{-1} E_{3}+\frac{\partial H_{2}}{\partial x_{1}}-\frac{\partial H_{1}}{\partial x_{2}}=0 .
\end{array}\right.
$$

By using the two first equations of $(13)$, we eliminate the radial components $H_{1}$ and $E_{1}$ to get

$$
\left\{\begin{aligned}
-i k Z_{0} H_{2}-\frac{\partial E_{3}}{\partial x_{1}}+\frac{Z_{0}}{i k}\left(\frac{\partial^{2} H_{2}}{\partial x_{3}^{2}}-\frac{\partial^{2} H_{3}}{\partial x_{2} \partial x_{3}}\right) & =0, \\
-i k Z_{0} H_{3}+\frac{\partial E_{2}}{\partial x_{1}}+\frac{Z_{0}}{i k}\left(\frac{\partial^{2} H_{3}}{\partial x_{2}^{2}}-\frac{\partial^{2} H_{2}}{\partial x_{2} \partial x_{3}}\right) & =0 \\
i k Z_{0}^{-1} E_{2}-\frac{\partial H_{3}}{\partial x_{1}}+\frac{1}{i k Z_{0}}\left(\frac{\partial^{2} E_{3}}{\partial x_{3} \partial x_{2}}-\frac{\partial^{2} E_{2}}{\partial x_{3}^{2}}\right) & =0 \\
i k Z_{0}^{-1} E_{3}+\frac{\partial H_{2}}{\partial x_{1}}+\frac{1}{i k Z_{0}}\left(\frac{\partial^{2} E_{2}}{\partial x_{2} \partial x_{3}}-\frac{\partial^{2} E_{3}}{\partial x_{2}^{2}}\right) & =0 .
\end{aligned}\right.
$$


The application of the partial Fourier transform along $\mathbf{x}_{\|}$to system (14) leads to

$$
\partial_{x_{1}}\left(\begin{array}{c}
\mathbf{n} \times(\widehat{\mathbf{E}} \times \mathbf{n}) \\
\mathbf{n} \times(\widehat{\mathbf{H}} \times \mathbf{n})
\end{array}\right)-\imath \lambda\left(\begin{array}{cc}
0 & \mathcal{M} \\
\mathcal{M}^{-1} & 0
\end{array}\right)\left(\begin{array}{c}
\mathbf{n} \times(\widehat{\mathbf{E}} \times \mathbf{n}) \\
\mathbf{n} \times(\widehat{\mathbf{H}} \times \mathbf{n})
\end{array}\right)=\left(\begin{array}{l}
\mathbf{0} \\
\mathbf{0}
\end{array}\right)
$$

with

$$
\mathcal{M}:=Z_{0} \lambda^{-1}\left(\begin{array}{cc}
\xi_{2} \xi_{3} & \left(k^{2}-\xi_{2}^{2}\right) \\
-\left(k^{2}-\xi_{3}^{2}\right) & -\xi_{2} \xi_{3}
\end{array}\right)
$$

The eigenvalues of the matrix $M$ that defines system (15) are -1 and +1 , with multiplicity two. The associated eigenvectors are respectively

$$
\mathbf{v}_{1}^{-}=\left(-\frac{Z_{0}\left(k^{2}-\xi_{2}^{2}\right)}{\lambda}, \frac{Z_{0} \xi_{2} \xi_{3}}{\lambda}, 0,1\right)^{t}, \quad \mathbf{v}_{2}^{-}=\left(-\frac{Z_{0} \xi_{2} \xi_{3}}{\lambda}, \frac{Z_{0}\left(k^{2}-\xi_{3}^{2}\right)}{\lambda}, 1,0\right)^{t}
$$

and

$$
\mathbf{v}_{1}^{+}=\left(\frac{Z_{0} \xi_{2} \xi_{3}}{\lambda},-\frac{Z_{0}\left(k^{2}-\xi_{3}^{2}\right)}{\lambda}, 1,0\right)^{t}, \quad \mathbf{v}_{2}^{+}=\left(\frac{Z_{0}\left(k^{2}-\xi_{2}^{2}\right)}{\lambda},-\frac{Z_{0} \xi_{2} \xi_{3}}{\lambda}, 0,1\right)^{t},
$$

where $\lambda=k \sqrt{k^{2}-\|\boldsymbol{\xi}\|^{2}}$. The matrix $M$ can be diagonalized as $M=P D P^{-1}$, with $D=$ $\operatorname{diag}(-1,-1,+1,+1)$ and $P=\left(\mathbf{v}_{1}, \mathbf{v}_{2}, \mathbf{v}_{3}, \mathbf{v}_{4}\right)$. Another way of writing $P$ is the following

$$
P:=\left(\begin{array}{cc}
-\mathcal{S} \mathcal{M} & \mathcal{M} \\
\mathcal{S} & \mathcal{I}
\end{array}\right)
$$

where $\mathcal{S}:=(0,1 ; 1,0)$ is the symmetry according to the main diagonal in the two-dimensional plane. The inverse of $P$ is

$$
P^{-1}:=\frac{1}{2}\left(\begin{array}{cc}
Z_{0}^{-2} \mathcal{S M} & \mathcal{S} \\
-Z_{0}^{-2} \mathcal{M} & \mathcal{I}
\end{array}\right)
$$

This means that we can also rewrite system (15) as a first-order hyperbolic system

$$
\partial_{x_{1}} \mathbf{W}-\imath \lambda D \mathbf{W}=\mathbf{0}, \quad \text { for } x_{1} \geq 0,
$$

where $\mathbf{W}=P^{-1} \mathbf{U}, \mathbf{U}=(\mathbf{n} \times(\widehat{\mathbf{E}} \times \mathbf{n}), \mathbf{n} \times(\widehat{\mathbf{H}} \times \mathbf{n}))^{t}$. The solution of Eq. 20 is: $\mathbf{W}\left(x_{1}\right)=$ $e^{\imath \lambda D x_{1}} \mathbf{W}(0)$. Since we are characterizing the part of the wave field that is square integrable in the right half-space, then we must impose that: $\mathbf{W}_{1,2}=\mathbf{0}$. Coming back to $\mathbf{U}$, we obtain the equation

$$
Z_{0}^{-2} \mathcal{S M}(\mathbf{n} \times(\widehat{\mathbf{E}} \times \mathbf{n}))+\mathcal{S}(\mathbf{n} \times(\widehat{\mathbf{H}} \times \mathbf{n}))=\mathbf{0},
$$

for $x_{1} \geq 0$. In addition, we have

$$
Z_{0}^{-2} \mathcal{S M}=\frac{1}{Z_{0}} \lambda^{-1}\left(\left(\begin{array}{cc}
k^{2} & 0 \\
0 & k^{2}
\end{array}\right)-\left(\begin{array}{cc}
\xi_{3}^{2} & -\xi_{2} \xi_{3} \\
-\xi_{2} \xi_{3} & \xi_{2}^{2}
\end{array}\right)\right)
$$

Since $\lambda=k \sqrt{k^{2}-\|\boldsymbol{\xi}\|^{2}}=k^{2} \sqrt{1-\frac{\|\boldsymbol{\xi}\|^{2}}{k^{2}}}$, applying an inverse Fourier transform along $\boldsymbol{\xi}$ leads to the exact operator

$$
\boldsymbol{\Lambda}^{\mathrm{ex}}=Z_{0}^{-1}\left(\mathbf{I}+\frac{\boldsymbol{\Delta}_{\Gamma}}{k^{2}}\right)^{-\frac{1}{2}}\left(\mathbf{I}-\frac{1}{k^{2}} \operatorname{curl}_{\Gamma} \operatorname{curl}_{\Gamma}\right) .
$$

Finally, we obtain the MtE equation

$$
\mathbf{M}+\boldsymbol{\Lambda}^{\mathrm{ex}}(\mathbf{n} \times \mathbf{J})=0 .
$$


Now, let us assume that $\Gamma$ is a general smooth surface. We propose a formal way of constructing a first version of the surface operator (11)-(12) in this case. Let us consider that, at a point $\mathbf{x}_{0}$ of the surface $\Gamma$, we make a tangent plane approximation of the map that links $\mathbf{M}$ to $\mathbf{J}$. This means that, locally, the curved surface at $\mathbf{x}_{0}$ is replaced by the local tangent plane $T_{\mathbf{x}_{0}}(\Gamma)$. As a first approximation, we can consider that (11)-(12) is valid, the extension of the various surface tangential operators being direct from their definition (see subsection 3.1). Following this approach, we formally deduce the following surface approximation onto $\Gamma$

$$
\mathbf{M}+\boldsymbol{\Lambda}(\mathbf{n} \times \mathbf{J})=0, \text { on } \Gamma,
$$

where the operator $\boldsymbol{\Lambda}$ is defined by

$$
\boldsymbol{\Lambda}=\boldsymbol{\Lambda}_{1}^{-1} \boldsymbol{\Lambda}_{2}, \quad \boldsymbol{\Lambda}_{1}=Z_{0}\left(\mathbf{I}+\frac{\boldsymbol{\Delta}_{\Gamma}}{k^{2}}\right)^{\frac{1}{2}}, \quad \boldsymbol{\Lambda}_{2}=\mathbf{I}-\frac{1}{k^{2}} \operatorname{curl}_{\Gamma} \operatorname{curl}_{\Gamma}
$$

Following this approach, this means that $x_{1}$ is the local radial variable along the outwardly directed unit normal vector $\mathbf{n}\left(\mathbf{x}_{0}\right)$, and $\mathbf{x}_{\|}$is the tangential variable in the tangent plane $T_{\mathbf{x}_{0}}(\Gamma)$.

A more rigorous approach, which would lead to the same conclusion, is the following. First, instead of considering the tangent plane approximation, local coordinate mappings based on a partition of unity of $\Gamma$ allow to set the initial system (1) into a general local system of coordinates. As a result, the system can be written as a new system set in the half-space, the radial variable along $\mathbf{n}\left(\mathbf{x}_{0}\right)$ now playing the role of $x_{1}$. The price to pay is that the system is no longer a constant coefficients system like for (13). Hence, the diagonalization in the Fourier space of the resulting system cannot be made in an exact way. Nevertheless, the technique of microlocal diagonalization for hyperbolic systems, initiated by Majda \& Osher [43] and applied in the background of Absorbing Boundary Conditions by Engquist and Majda [32, can be used. Doing that, this rigorous way of proceeding leads to a first-order approximation that strictly corresponds to (23)-(24) since, indeed, the curvatures are considered as zero. The strenght of this technique is that higher-order corrective terms can be computed through the diagonalization process making use of the pseudodifferential operator calculus. For example, the next approximation includes curvature tensor effects. However, as shown below, we propose another improvement of $(23)-(24)$ that also formally includes curvature terms. Even if this second approach appears as less rigorous mathematically, it has also been proven to be much more accurate in practical computations for the Helmholtz equation [14].

Let us remark that $(23)-(24)$ is not the only boundary condition that can be built. Indeed, another solution that is studied later is to write

$$
\boldsymbol{\Lambda}_{1} \mathbf{M}+\boldsymbol{\Lambda}_{2}(\mathbf{n} \times \mathbf{J})=0, \text { on } \Gamma .
$$

We will see during the approximation procedure and the numerical experiments the difference between these two possibilities. All these relations fall into the framework of expressions like (7).

\subsection{Construction of nonlocal approximate MtE operators: accuracy improve- ment by regularization}

One way to assess the accuracy of the approximate OSRC operator (24) is to directly apply it on the surface of a perfectly conducting sphere $\Gamma:=\mathbb{S}_{R}$ of radius $R>0$. This situation allows us to understand analytically where some errors can appear in the numerical solution and provides a guideline to build more accurate operators.

Let us consider that the sphere is illuminated by a plane wave traveling in the $x_{3}$-direction, i.e. $\mathbf{E}^{\text {inc }}(\mathbf{x})=(1,0,0)^{t} e^{-i k x_{3}}$. By using a decomposition in the Debye potentials, one gets the following 
expression of the tangential trace of $\mathbf{E}^{\text {inc }}$ onto $\mathbb{S}_{R}$

$$
\begin{aligned}
\mathbf{E}_{T}^{\text {inc }}(R \mathbf{n})= & -\frac{1}{k} \sum_{m=1}^{\infty}(-\imath)^{m} \frac{2 m+1}{m(m+1)} \psi_{m}(k R) \mathbf{n} \times \nabla_{\mathbb{S}_{R}}\left(P_{n}^{1}(\cos (\theta)) \sin (\phi)\right) \\
& +\frac{1}{k} \sum_{m=1}^{\infty}(-\imath)^{m} \frac{2 m+1}{m(m+1)} \imath \psi_{m}^{\prime}(k R) \nabla_{\mathbb{S}_{R}}\left(P_{n}^{1}(\cos (\theta)) \cos (\phi)\right)
\end{aligned}
$$

where $\psi_{m}$ is the Ricatti-Bessel function of order $m, P_{m}^{1}$ is the first-order Legendre function of degree $m$ and $(\theta, \phi)$ are the spherical coordinates. In this context, $\mathbf{M}$ is explicitly given by

$$
\mathbf{M}=\frac{Z_{0}^{-1}}{k} \sum_{m=1}^{\infty} A_{1, m}^{\text {exa }} \mathbf{n} \times \nabla_{\mathbb{S}_{R}}\left(P_{n}^{1}(\cos (\theta)) \sin (\phi)\right)-\frac{Z_{0}^{-1}}{k} \sum_{m=1}^{\infty} A_{2, m}^{\text {exa }} \nabla_{\mathbb{S}_{R}}\left(P_{n}^{1}(\cos (\theta)) \cos (\phi)\right),
$$

where

$$
\left\{\begin{array}{c}
A_{1, m}^{\mathrm{exa}}=-(-\imath)^{m+1} \frac{2 m+1}{m(m+1)} \frac{\xi_{m}^{(1)^{\prime}}(k R)}{\xi_{m}^{(1)}(k R)} \psi_{m}(k R), \\
A_{2, m}^{\mathrm{exa}}=(-\imath)^{m} \frac{2 m+1}{m(m+1)} \frac{\xi_{m}^{(1)}(k R)}{\xi_{m}^{(1)^{\prime}}(k R)} \psi_{m}^{\prime}(k R),
\end{array}\right.
$$

and $\xi_{m}^{(1)}$ designates the first-kind spherical Hankel's function of order $m$.

Now, by using the plane wave expansion given by (26), we can also compute the approximate surface current $\widetilde{\mathbf{M}}$ corresponding to the OSRC equation associated to $(23)-(24)$ as

$$
\widetilde{\mathbf{M}}=\frac{Z_{0}^{-1}}{k} \sum_{m=1}^{\infty} A_{1, m}^{\mathrm{app}} \mathbf{n} \times \nabla_{\mathbb{S}_{R}}\left(P_{n}^{1}(\cos (\theta)) \sin (\phi)\right)-\frac{Z_{0}^{-1}}{k} \sum_{m=1}^{\infty} A_{2, m}^{\mathrm{app}} \nabla_{\mathbb{S}_{R}}\left(P_{n}^{1}(\cos (\theta)) \cos (\phi)\right),
$$

where

$$
\left\{\begin{array}{l}
A_{1, m}^{\mathrm{app}}=\imath(-\imath)^{m+1} \frac{2 m+1}{m(m+1)}\left(1-\frac{m(m+1)}{k^{2} R^{2}}\right)^{\frac{1}{2}} \psi_{m}(k R), \\
A_{2, m}^{\mathrm{app}}=\imath(-\imath)^{m} \frac{2 m+1}{m(m+1)}\left(1-\frac{m(m+1)}{k^{2} R^{2}}\right)^{-\frac{1}{2}} \psi_{m}^{\prime}(k R) .
\end{array}\right.
$$

The expressions (29)-(30) are obtained by using the following properties (see [48] p.39)

$$
\left\{\begin{aligned}
\boldsymbol{\Delta}_{\mathbb{S}_{R}} \nabla_{\mathbb{S}_{R}} Y_{m}^{n} & =-\frac{m(m+1)}{R^{2}} \nabla_{\mathbb{S}_{R}} Y_{m}^{n} \\
\boldsymbol{\Delta}_{\mathbb{S}_{R}} \operatorname{curl}_{\mathbb{S}_{R}} Y_{m}^{n} & =-\frac{m(m+1)}{R^{2}} \operatorname{curl}_{\mathbb{S}_{R}} Y_{m}^{n}
\end{aligned}\right.
$$

where $Y_{m}^{n}$ is the spherical harmonic of order $(m, n)$.

We report on Figure 1 the real parts of the coefficients $A_{i, m}^{\text {exa }}$ and $A_{i, m}^{\text {app }}$, for $i=1,2$, with respect to the modes $m$ (similar curves can be obtained for the imaginary parts). We fix $k=50$ and $R=1$. We can observe a very good agreement between the exact and approximate coefficients for both the propagative $(1 \leq m \ll k R)$ and evanescent $(m \gg k R)$ modes. However, some errors are localized for the grazing modes $m \simeq k R$ near the cut-off frequency. This is indeed due to the singularity that arises in the square-root in (30).

To improve the accuracy for these frequencies and avoid the singularity, we regularize the squareroot operator by adding a small damping parameter $\varepsilon>0$ to the wavenumber $k$ that appears inside the square-root. Let us set $k_{\varepsilon}=k+\imath \varepsilon$ and introduce the new MtE operator

$$
\mathbf{M}+\boldsymbol{\Lambda}_{\varepsilon}(\mathbf{n} \times \mathbf{J})=0, \text { on } \Gamma,
$$



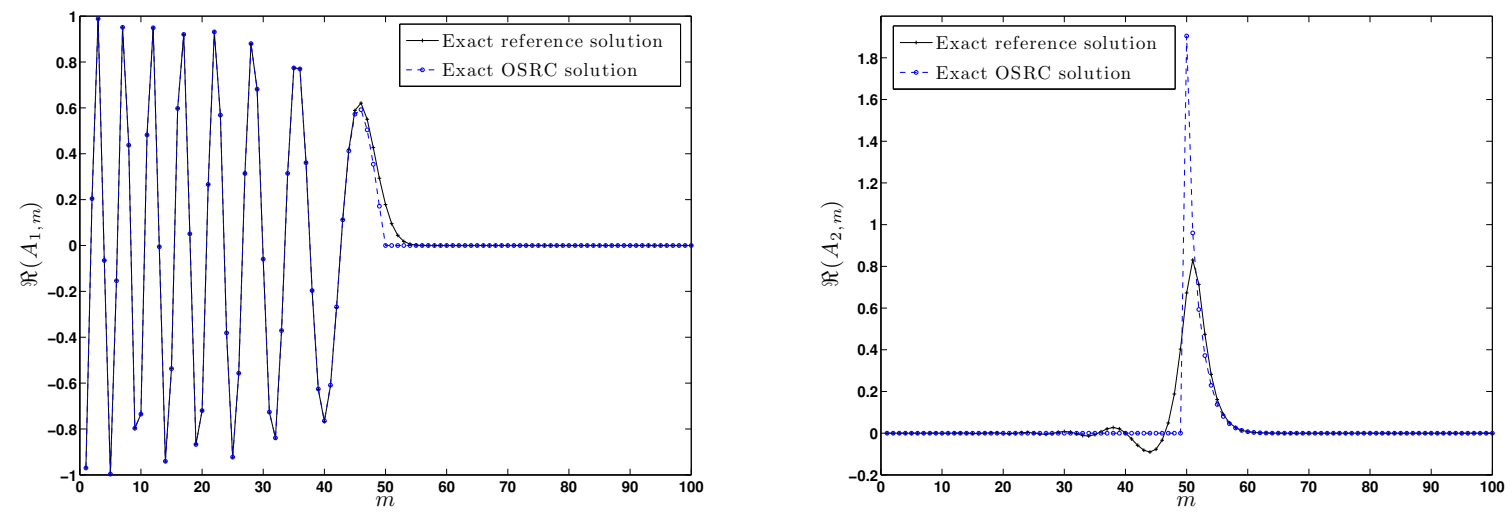

Figure 1: Real parts of the coefficients $A_{i, m}^{\text {exa }}$ and $A_{i, m}^{\text {app }}, i=1,2(k=50$ and $R=1)$.

where we define the regularized operator $\boldsymbol{\Lambda}_{\varepsilon}$ by

$$
\boldsymbol{\Lambda}_{\varepsilon}=\boldsymbol{\Lambda}_{1, \varepsilon}^{-1} \boldsymbol{\Lambda}_{2, \varepsilon}, \quad \boldsymbol{\Lambda}_{1, \varepsilon}=Z_{0}\left(\mathbf{I}+\frac{1}{k_{\varepsilon}^{2}} \boldsymbol{\Delta}_{\Gamma}\right)^{\frac{1}{2}}, \quad \boldsymbol{\Lambda}_{2, \varepsilon}=\mathbf{I}-\frac{1}{k_{\varepsilon}^{2}} \operatorname{curl}_{\Gamma} \operatorname{curl}_{\Gamma} .
$$

Let us remark that it is also important to add the damping term in the operator $\boldsymbol{\Lambda}_{2, \varepsilon}$ to avoid some possible resonant frequencies and have a corresponding well-posed problem. Numerical simulations confirm this point. Considering this new OSRC, we can compute the associated coefficients $A_{i, m, \varepsilon}^{\text {app }}$, $i=1,2$. To fix the value of $\varepsilon$, we propose to minimize the error between the exact and approximate coefficients, that is $A_{1, m}^{\text {exa }}$ and $A_{1, m, \varepsilon}^{\text {app }}$, for $m \geq 1$ and $\varepsilon>0$. Let $\rho$ be the function defined by

$$
\rho_{m, \varepsilon}:=\left|A_{1, m}^{\mathrm{exa}}-A_{1, m, \varepsilon}^{\mathrm{app}}\right| .
$$

We consider the following min-max problem

$$
\left\{\begin{array}{l}
\text { Find }\left(\varepsilon_{\mathrm{opt}}, m_{\mathrm{opt}}\right) \text { solution to } \\
\rho_{\varepsilon_{\mathrm{opt}}, m_{\mathrm{opt}}}:=\min _{\varepsilon>0} \max _{m \geq 1} \rho_{m, \varepsilon}
\end{array}\right.
$$

Then, we have the following proposition.

Proposition 2. For $k R$ sufficiently large, an approximation of $\varepsilon_{\text {opt }}$ (that appears in the solution of the min-max problem (35)) is given by

$$
\varepsilon_{o p t} \simeq 0.39 k^{1 / 3} R^{-2 / 3} .
$$

Proof. The function $\rho_{\varepsilon, m}$ is defined by

$$
\rho_{\varepsilon, m}:=\left|\left(1-\frac{m(m+1)}{R^{2} k_{\varepsilon}^{2}}\right)^{\frac{1}{2}}+\frac{\xi_{m}^{(1)^{\prime}}(k R)}{\xi_{m}^{(1)}(k R)}\right|\left|\psi_{m}(k R)\right| .
$$

For a fixed $\varepsilon$, we numerically observe that the error is maximal at $m=[k R]$, where $[\cdot]$ denotes the integer part of a real number (for $k R$ large enough (see Figure 2) ).

By taking $m_{\mathrm{opt}}=[k R]$, we have to solve the corresponding minimization problem

$$
\left\{\begin{array}{l}
\text { Find } \varepsilon_{\mathrm{opt}} \text { solution to } \\
\rho_{\varepsilon_{\mathrm{opt}},[k R]}:=\min _{\varepsilon>0} \rho_{\varepsilon,[k R]} .
\end{array}\right.
$$




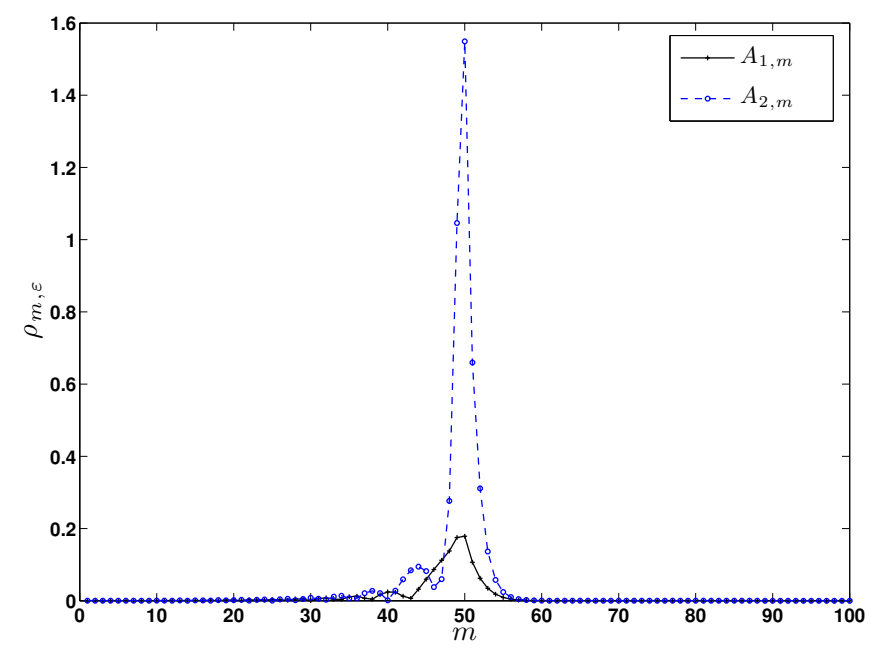

Figure 2: Variations of the error $\rho_{m, \varepsilon}$ with respect to $m$ (for $\left.\varepsilon=0\right)(k=50$ and $R=1)$.

To simplify the computations, let us assume that $k$ and $R$ are two integers. If we set $K=k R$, then we have

$$
\begin{aligned}
& \psi_{K}(K)=\sqrt{\frac{\pi K}{2}} J_{K+\frac{1}{2}}(K), \quad \xi_{K}^{(1)}(K)=\sqrt{\frac{\pi K}{2}} H_{K+\frac{1}{2}}^{(1)}(K), \\
& \xi_{K}^{(1)^{\prime}}(K)=\sqrt{\frac{\pi K}{2}}\left(\frac{K+1}{2 K+1} H_{K-\frac{1}{2}}^{(1)}(K)-\frac{K}{2 K+1} H_{K+\frac{3}{2}}^{(1)}(K)\right),
\end{aligned}
$$

with $H_{K}^{(1)}(x)=J_{K}(x)+\imath Y_{K}(x)$. From [1] (pp. 367), we have for $\mu$ large

$$
J_{\mu}\left(\mu+z \mu^{\frac{1}{3}}\right) \sim \frac{2^{\frac{1}{3}}}{\mu^{\frac{1}{3}}} A i\left(-2^{\frac{1}{3}} z\right), \quad Y_{\mu}\left(\mu+z \mu^{\frac{1}{3}}\right) \sim-\frac{2^{\frac{1}{3}}}{\mu^{\frac{1}{3}}} B i\left(-2^{\frac{1}{3}} z\right),
$$

where $A i$ and $B i$ are the Airy functions. For $\mu=K+\frac{1}{2}$ and $z=-\frac{\left(K+\frac{1}{2}\right)^{-\frac{1}{3}}}{2}$, we get

$$
\left\{\begin{array}{l}
J_{K+\frac{1}{2}}(K) \sim 2^{\frac{1}{3}}\left(K+\frac{1}{2}\right)^{-1 / 3} A_{i}\left(2^{\frac{1}{3}}\left(K+\frac{1}{2}\right)^{-1 / 3}\right), \\
Y_{K+\frac{1}{2}}(K) \sim-2^{\frac{1}{3}}\left(K+\frac{1}{2}\right)^{-1 / 3} B_{i}\left(-2^{-\frac{5}{3}}\left(K+\frac{1}{2}\right)^{-1 / 3}\right) .
\end{array}\right.
$$

For a small argument $z=-\frac{\left(K+\frac{1}{2}\right)^{-\frac{1}{3}}}{2}$, a Taylor's expansion leads to: $A i(z) \sim A i(0)+A i^{\prime}(0) z=a+b z$ and $B i(z) \sim B i(0)+B i^{\prime}(0) z=\sqrt{3}(a-b z)$, where $a$ and $b$ are computed through the Gamma function: $a=\simeq 0.355, b \simeq-0.259$. By using these approximations, one obtains for $K \rightarrow \infty$

$$
\left\{\begin{array}{rlrl}
\psi_{K}(K) & \sim \frac{a \sqrt{\pi}}{2^{\frac{1}{6}}} K^{\frac{1}{6}}, & & \xi_{K}^{(1)}(K) \sim \frac{a \sqrt{\pi}(1-\imath \sqrt{3})}{2^{\frac{1}{6}}} K^{\frac{1}{6}}, \\
\xi_{K}^{(1)^{\prime}}(K) \sim \frac{b \sqrt{\pi}(1+\imath \sqrt{3})}{K^{\frac{1}{6}}} 2^{\frac{1}{6}}, & & \left(1-\frac{K(K+1)}{R^{2} k^{2}}\right)^{\frac{1}{2}} \sim \sqrt{2 \imath \varepsilon R-1} K^{-\frac{1}{2}} .
\end{array}\right.
$$

Combining these approximations with (37) for $m_{\mathrm{opt}}=K$, we get the following approximation

$$
\rho_{\varepsilon, K}^{\prime}(\varepsilon) \sim 64 b^{4} R^{2} \varepsilon^{2}+512 \sqrt{3} \sqrt[3]{2} a^{2} K^{-2 / 3} R^{3} \varepsilon^{3}-1024 \sqrt[3]{4} a^{4} K^{-2 / 3} R^{4} \varepsilon^{4} .
$$

Deriving the above local expression provides the following approximation of the optimal value $\varepsilon_{\text {opt }}$ : $\varepsilon_{\mathrm{opt}}=(2+\sqrt{3}) 2^{-7 / 3} a^{-2} b^{2} k^{1 / 3} R^{-2 / 3} \simeq 0.39 k^{1 / 3} R^{-2 / 3}$. 
For comparison, we report on Figure 3 the real parts of the coefficients $A_{i, m}^{\text {exa }}$ and $A_{i, m, \varepsilon_{\mathrm{opt}}}^{\text {app }}, i=1$, 2. We can see that we have a very good agreement between the exact and approximate coefficients considering the optimal damping parameter $\varepsilon_{\mathrm{opt}}$. We obtain similar results for the imaginary parts.
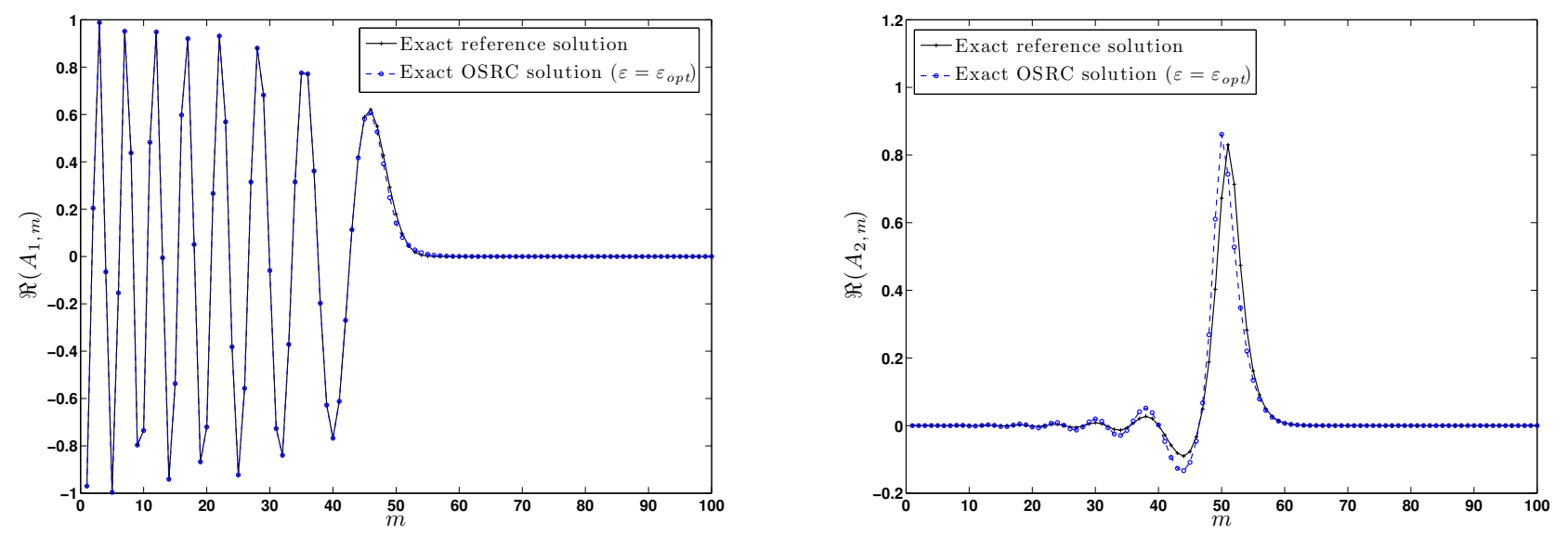

Figure 3: Real parts of the coefficients $A_{i, m}^{\text {exa }}$ and $A_{i, m, \varepsilon_{\mathrm{opt}}}^{\text {app }}, i=1,2(k=50$ and $R=1)$.

All the computations during the estimate of $\varepsilon_{\text {opt }}$ have been done in the special case of a sphere. The extension to a more general shape is a priori not trivial. Indeed, the radius $R$ in the case of the sphere can be seen as the simplification of different geometrical quantities in the general framework (Gauss curvature, mean curvature,...). We make the choice here to replace $1 / R$ by $\mathcal{H}$ which is the mean curvature at the boundary, e.g. the average of the two principal curvatures at the boundary. This implies that the corresponding damping parameter is now

$$
\varepsilon_{\text {opt }}=0.39 k^{1 / 3} \mathcal{H}^{2 / 3}
$$

in (32)-(33). However, if we do this formal substitution and keep on developing the localization process of the operators $\boldsymbol{\Lambda}_{1, \varepsilon_{\mathrm{opt}}}$ and $\boldsymbol{\Lambda}_{2, \varepsilon_{\mathrm{opt}}}$ as in Section 4.1, it can be seen that the associated variational formulations are non symmetrical (we do not detail the calculations, which are left to the reader). In fact, a better adapted way of deriving the regularized MtE operator is to consider the following symmetrical expression

$$
\mathbf{M}+\boldsymbol{\Lambda}_{\varepsilon_{\mathrm{opt}}}(\mathbf{n} \times \mathbf{J})=0, \text { on } \Gamma,
$$

with

$$
\begin{aligned}
& \boldsymbol{\Lambda}_{\varepsilon_{\mathrm{opt}}}=\boldsymbol{\Lambda}_{1, \varepsilon_{\mathrm{opt}}}^{-1} \boldsymbol{\Lambda}_{2, \varepsilon_{\mathrm{opt}}}, \quad \boldsymbol{\Lambda}_{1, \varepsilon_{\mathrm{opt}}}=Z_{0}\left(\mathbf{I}+\nabla_{\Gamma} \frac{1}{k_{\varepsilon_{\mathrm{opt}}}^{2}} \operatorname{div}_{\Gamma}-\operatorname{curl}_{\Gamma} \frac{1}{k_{\varepsilon_{\mathrm{opt}}}^{2}} \operatorname{curl}_{\Gamma}\right)^{1 / 2}, \\
& \boldsymbol{\Lambda}_{2, \varepsilon_{\mathrm{opt}}}=\mathbf{I}-\operatorname{curl}_{\Gamma} \frac{1}{k_{\varepsilon_{\mathrm{opt}}}^{2}} \operatorname{curl}_{\Gamma},
\end{aligned}
$$

for a general arbitrarily-shaped boundary $\Gamma$. For conciseness, we do not precise the "opt" index in $\varepsilon_{\text {opt }}$ defined by relation (43) and write $\varepsilon$ in the sequel of the paper.

\section{Localization and discretization of the MtE operators}

\subsection{Local representation of the MtE operators}

We now consider that we apply the MtE operator as an On-Surface Radiation Condition (OSRC) 6, 38. This means that we solve approximately the boundary-value problem (1) by setting one of the 
MtE operators on the physical surface $\Gamma$, and, with the help of the PEC boundary condition in (1), we solve the scattering problem. Thanks to (5)-(6), we get an approximation of the electromagnetic far-field pattern.

From (1), 44) and (45), we can get an OSRC approximation of $\mathbf{M}$ through the equation

$$
\boldsymbol{\Lambda}_{1, \varepsilon} \mathbf{M}=\boldsymbol{\Lambda}_{2, \varepsilon}\left(\mathbf{n} \times \mathbf{J}^{\text {inc }}\right) \text {, on } \Gamma .
$$

The operator $\boldsymbol{\Lambda}_{2, \varepsilon}$ given by (45) is a local symmetrical operator that can be approximated quite directly. The difficulty when one wants to numerically solve 46 is more related to the operator $\boldsymbol{\Lambda}_{1, \varepsilon}^{-1}$ which is a nonlocal pseudodifferential operator. More precisely, let us define the following local symmetrical surface operator

$$
\mathscr{T}:=\nabla_{\Gamma} \frac{1}{k_{\varepsilon}^{2}} \operatorname{div}_{\Gamma}-\operatorname{curl}_{\Gamma} \frac{1}{k_{\varepsilon}^{2}} \operatorname{curl}_{\Gamma}
$$

Then, we have

$$
\Lambda_{1, \varepsilon}:=Z_{0}(\mathbf{I}+\mathscr{T})^{1 / 2} .
$$

Discretizing such a square-root operator is non trivial, most particularly for a general surface $\Gamma$. Furthermore, even in the positive case and like for integral equation formulations, the resulting discrete matrix representation would lead to solving an associated full linear system since the operator is nonlocal. One usual way to obtain a local representation and a sparse discrete matrix representation is to approximate the square-root function by rational approximations [14]. A rotating branch-cut rational Padé approximation of the square-root function [45] is given by

$$
(1+z)^{1 / 2} \approx e^{\imath \frac{\theta_{p}}{2}} R_{N_{p}}\left((1+z) e^{-\imath \theta_{p}}-1\right)=C_{0}+\sum_{j=1}^{N_{p}} \frac{A_{j} z}{1+B_{j} z}=R_{0}-\sum_{j=1}^{N_{p}} \frac{A_{j}}{B_{j}\left(1+B_{j} z\right)},
$$

where $R_{N_{p}}$ is the standard real-valued Padé approximation of order $N_{p}$

$$
(1+z)^{1 / 2} \approx R_{N_{p}}(z)=1+\sum_{j=1}^{N_{p}} \frac{a_{j} z}{1+b_{j} z}
$$

with

$$
a_{j}=\frac{2}{2 N_{p}+1} \sin ^{2}\left(\frac{j \pi}{2 N_{p}+1}\right), \quad b_{j}=\cos ^{2}\left(\frac{j \pi}{2 N_{p}+1}\right) .
$$

The angle of rotation $\theta_{p}$ is a free parameter that has to be fixed during the numerical simulations and the constants are given by

$$
\begin{aligned}
C_{0} & =e^{\imath \frac{\theta_{p}}{2}} R_{N_{p}}\left(e^{-\imath \theta_{p}}-1\right), & A_{j} & =\frac{e^{-\imath \frac{\theta_{p}}{2}} a_{j}}{\left(1+b_{j}\left(e^{-\imath \theta_{p}}-1\right)\right)^{2}}, \\
B_{j} & =\frac{e^{-\imath \theta_{p}} b_{j}}{1+b_{j}\left(e^{-\imath \theta_{p}}-1\right)}, & R_{0} & =C_{0}+\sum_{j=1}^{N_{p}} \frac{A_{j}}{B_{j}}
\end{aligned}
$$

Formally (considering that $z=\mathscr{T}$ ), we approximate $\boldsymbol{\Lambda}_{1, \varepsilon}$ by

$$
\boldsymbol{\Lambda}_{1, \varepsilon}=Z_{0}(\mathbf{I}+\mathscr{T})^{1 / 2} \approx Z_{0}\left(R_{0}-\sum_{j=1}^{N_{p}} \frac{A_{j}}{B_{j}}\left(\mathbf{I}+B_{j} \mathscr{T}\right)^{-1}\right) .
$$


Using this approximation, the expression (47) of $\mathscr{T}$ and introducing $N_{p}$ coupled auxiliary vector fields $\left\{\phi^{j}\right\}_{j=1, \ldots, N_{p}}$, one gets the local approximate representation of (46)

$$
\left\{\begin{array}{l}
\mathbf{M}-\sum_{j=1}^{N_{p}} \frac{A_{j}}{R_{0} B_{j}} \phi^{j}=-\frac{1}{Z_{0} R_{0}} \mathbf{u}, \quad \text { on } \Gamma \\
\mathbf{M}-\left(\mathbf{I}+B_{j}\left(\nabla_{\Gamma} \frac{1}{k_{\varepsilon}^{2}} \operatorname{div}_{\Gamma}-\operatorname{curl}_{\Gamma} \frac{1}{k_{\varepsilon}^{2}} \operatorname{curl}_{\Gamma}\right)\right) \phi^{j}=\mathbf{0}, j=1, . ., N_{p}, \quad \text { on } \Gamma
\end{array}\right.
$$

setting

$$
\mathbf{u}=-\left(\mathbf{I}-\operatorname{curl}_{\Gamma} \frac{1}{k_{\varepsilon}^{2}} \operatorname{curl}_{\Gamma}\right)\left(\mathbf{n} \times \mathbf{J}^{\mathrm{inc}}\right) .
$$

Another possibility is to rather use a formulation based on

$$
\mathbf{M}=\boldsymbol{\Lambda}_{1, \varepsilon}^{-1} \boldsymbol{\Lambda}_{2, \varepsilon}\left(\mathbf{n} \times \mathbf{J}^{\text {inc }}\right) \text {, on } \Gamma,
$$

with

$$
\boldsymbol{\Lambda}_{1, \varepsilon}^{-1}:=Z_{0}^{-1}(\mathbf{I}+\mathscr{T})^{-1 / 2}
$$

The approximation of $(1+z)^{-1 / 2}$ is realized in the same spirit as before. We use the fact that

$$
(1+z)^{1 / 2} \approx C_{0}+\sum_{j=1}^{N_{p}} \frac{A_{j} z}{1+B_{j} z}=\frac{P_{N_{p}}(z)}{Q_{N_{p}}(z)},
$$

where $P_{N_{p}}$ and $Q_{N_{p}}$ are two polynomials of degree $N_{p}$. As a consequence, the inverse of the square-root is approximated by

$$
(1+z)^{-\frac{1}{2}} \approx \frac{Q_{N_{p}}(z)}{P_{N_{p}}(z)}=r_{0}+\sum_{j=1}^{N_{p}} \frac{r_{j}}{z-q_{j}},
$$

where the coefficients $r_{0}, r_{j}$ and $q_{j}$ are obtained through the calculations of the roots of $P_{N_{p}}$ and $Q_{N_{p}}$, and next decomposing the fraction into elementary elements. Therefore, by using (57), the OSRC (55)-(56) is computed by

$$
\left\{\begin{array}{l}
\mathbf{M}+\sum_{j=1}^{N_{p}} \frac{r_{j}}{Z_{0}} \boldsymbol{\phi}^{j}=-\frac{r_{0}}{Z_{0}} \mathbf{u} \\
\left(\nabla_{\Gamma} \frac{1}{k_{\varepsilon}^{2}} \operatorname{div}_{\Gamma}-\operatorname{curl}_{\Gamma} \frac{1}{k_{\varepsilon}^{2}} \operatorname{curl}_{\Gamma}-q_{j} \mathbf{I}\right) \phi^{j}=\mathbf{u}, \quad j=1, \ldots, N_{p},
\end{array}\right.
$$

where $\mathbf{u}$ is given by (54). The nice point with (58) is that there is no coupling between the auxiliary vector fields $\phi^{j}, j=1, \ldots, N_{p}$. This is not the case when considering system (53). During the numerical simulations, we will see that this directly impacts the overall computational performances of the methods (see Table 3).

Concerning the choice of the angle of rotation, one possibility consists in writing the exact solution $\widetilde{\mathbf{M}}^{\text {Pade }}$ for the scattering by the sphere $\mathbb{S}_{R}$ and for the Padé approximation (58). Some calculations lead to the expression

$$
\begin{array}{r}
\widetilde{\mathbf{M}}^{\text {Pade }}=\frac{Z_{0}^{-1}}{k} \sum_{m=1}^{\infty} A_{1, m}^{\text {Pade }} \mathbf{n} \times \nabla_{\mathbb{S}_{R}}\left(P_{n}^{1}(\cos (\theta)) \sin (\phi)\right) \\
-\frac{Z_{0}^{-1}}{k} \sum_{m=1}^{\infty} A_{2, m}^{\text {Pade }} \nabla_{\mathbb{S}_{R}}\left(P_{n}^{1}(\cos (\theta)) \cos (\phi)\right),
\end{array}
$$


where

$$
\left\{\begin{array}{l}
A_{1, m}^{\text {Pade }}=(-\imath)^{m+1} \frac{2 m+1}{m(m+1)}\left(1-\frac{m(m+1)}{k^{2} R^{2}}\right)\left(r_{0}+\sum_{j=1}^{N_{p}} \frac{r_{j}}{q_{j}} C_{m, j}\right) \psi_{m}(k R), \\
A_{2, m}^{\text {Pade }}=\imath(-\imath)^{m} \frac{2 m+1}{m(m+1)}\left(r_{0}-\sum_{j=1}^{N_{p}} \frac{r_{j}}{q_{j}}\left(1+\frac{m(m+1)}{q_{j} k^{2} R^{2}}\right)^{-1}\right) \psi_{m}^{\prime}(k R),
\end{array}\right.
$$

with

$$
C_{m, j}=\left(1-\frac{m(m+1)}{k^{2} R^{2}}\right)\left(1+\frac{m(m+1)}{q_{j} k^{2} R^{2}}\right)^{-1} .
$$

We can assess the accuracy of the Padé approximation, according to $N_{p}$ and $\theta_{p}$, by computing $\left\|\mathbf{A}_{2}^{\text {app }}-\mathbf{A}_{2}^{\text {Pade }}\right\|(\|\cdot\|$ is the euclidian norm $)$, with $\mathbf{A}_{2}^{\text {app }}:=\left(A_{2,1}^{\text {app }}, \ldots, A_{2, m_{\max }}^{\text {app }}\right)^{t}$ and $\mathbf{A}_{2}^{\text {Pade }}:=$ $\left(A_{2,1}^{\text {app }}, \ldots, A_{2, m_{\max }}^{\text {Pade }}\right)$. We report the numerical results in Table 1 , for $m_{\max }:=10[k]$ and $k=10$. We see that increasing $N_{p}$ leads to a better accuracy, but less trivially, considering $\theta_{p}=\pi / 2$ minimizes the error. Similar computations can be made by using the second formulation based on (56), the conclusion being the same. From now on, we choose $\theta_{p}=\pi / 2$.

\begin{tabular}{|c||c|c|c|c|}
\hline$\theta_{p}$ & $N_{p}=2$ & $N_{p}=4$ & $N_{p}=8$ & $N_{p}=16$ \\
\hline \hline 0 & $1.25 \times 10^{0}$ & $6.5 \times 10^{-1}$ & $3.7 \times 10^{-1}$ & $1.9 \times 10^{-1}$ \\
\hline$\frac{\pi}{8}$ & $3.6 \times 10^{-1}$ & $1.1 \times 10^{-1}$ & $1.4 \times 10^{-2}$ & $1.3 \times 10^{-3}$ \\
\hline$\frac{\pi}{6}$ & $2.7 \times 10^{-1}$ & $6.7 \times 10^{-2}$ & $5.0 \times 10^{-3}$ & $2.7 \times 10^{-4}$ \\
\hline$\frac{\pi}{4}$ & $1.6 \times 10^{-1}$ & $2.8 \times 10^{-2}$ & $9.4 \times 10^{-4}$ & $1.2 \times 10^{-5}$ \\
\hline$\frac{\pi}{3}$ & $1.1 \times 10^{-1}$ & $1.5 \times 10^{-2}$ & $3.2 \times 10^{-4}$ & $1.5 \times 10^{-6}$ \\
\hline$\frac{\pi}{2}$ & $8.0 \times 10^{-2}$ & $1.0 \times 10^{-2}$ & $1.7 \times 10^{-4}$ & $5.3 \times 10^{-7}$ \\
\hline$\frac{5 \pi}{8}$ & $1.2 \times 10^{-1}$ & $1.6 \times 10^{-2}$ & $4.6 \times 10^{-4}$ & $1.8 \times 10^{-5}$ \\
\hline$\frac{2 \pi}{3}$ & $1.4 \times 10^{-1}$ & $2.0 \times 10^{-2}$ & $5.4 \times 10^{-4}$ & $1.2 \times 10^{-5}$ \\
\hline$\frac{3 \pi}{4}$ & $2.1 \times 10^{-1}$ & $4.1 \times 10^{-2}$ & $1.7 \times 10^{-3}$ & $2.4 \times 10^{-5}$ \\
\hline
\end{tabular}

Table 1: $\left\|\mathbf{A}_{2}^{\text {app }}-\mathbf{A}_{2}^{\text {Pade }}\right\|_{2}$ vs. $N_{p}$ and $\theta_{p}$.

\subsection{Finite element discretization}

In view of a finite element approximation for a general shape $\Gamma$, we consider a polyhedral approximation $\Gamma_{h}$ of $\Gamma$, for a triangulation $\mathcal{T}_{h}:=\cup_{\ell=1}^{N_{T}} T^{\ell}$ involving $N_{T}$ surface triangles. Let us introduce: $\boldsymbol{\Phi}_{h}:=\left(\phi_{h}^{j}\right)_{j=1, \ldots, N_{p}}$ and $\boldsymbol{\rho}_{h}:=\left(\rho_{h}^{j}\right)_{j=1, \ldots, N_{p}}$. For solving system $(53)$, we use the following symmetrical weak formulation: find $\left(\mathbf{M}_{h}, \boldsymbol{\Phi}_{h}, \boldsymbol{\rho}_{h}\right) \in V_{h} \times V_{h}^{p} \times Z_{h}^{p}$ such that

$$
\left\{\begin{array}{c}
\int_{\Gamma_{h}} \mathbf{M}_{h} \cdot \mathbf{v}_{h} d \Gamma_{h}+\sum_{j=1}^{N_{p}} \alpha_{j} \int_{\Gamma_{h}} \phi_{h}^{j} \cdot \mathbf{v}_{h} d \Gamma_{h}=-\frac{1}{R_{0} Z_{0}} \int_{\Gamma_{h}} \mathbf{u}_{h} \cdot \mathbf{v}_{h} d \Gamma_{h}, \\
\int_{\Gamma_{h}} \mathbf{M}_{h} \cdot \mathbf{w}_{h}^{j} d \Gamma_{h}-\int_{\Gamma_{h}}\left(\phi_{h}^{j} \cdot \mathbf{w}_{h}^{j}-\frac{B_{j}}{k_{\varepsilon, h}^{2}} \operatorname{curl}_{\Gamma_{h}} \boldsymbol{\phi}_{h}^{j} \cdot \operatorname{curl}_{\Gamma_{h}} \mathbf{w}_{h}^{j}\right) d \Gamma_{h} \\
\quad-B_{j} \int_{\Gamma_{h}} \nabla_{\Gamma_{h}} \rho_{h}^{j} \cdot \mathbf{w}_{h}^{j} d \Gamma_{h}=0, \quad j=1, \ldots, N_{p}, \\
\int_{\Gamma_{h}}\left(k_{\varepsilon, h}^{2} \rho_{h}^{j} z_{h}^{j}+\phi_{h}^{j} \cdot \nabla_{\Gamma_{h}} z_{h}^{j}\right) d \Gamma_{h}=0, \quad j=1, \ldots, N_{p},
\end{array}\right.
$$


for any test-functions $\mathbf{v}_{h}$ and $\mathbf{w}_{h}^{j}$ in $V_{h}$, and $z_{h}^{j}$ in $Z_{h}$, for $j=1, \ldots, N_{p}$. The input vector $\mathbf{u}_{h}$ is computed through

$$
\int_{\Gamma_{h}} \mathbf{u}_{h} \cdot \mathbf{v}_{h} d \Gamma_{h}=\int_{\Gamma_{h}} \frac{1}{k_{\varepsilon, h}^{2}} \operatorname{curl}_{\Gamma_{h}}\left(\mathbf{n}_{h} \times \mathbf{J}_{h}^{\mathrm{inc}}\right) \cdot \operatorname{curl}_{\Gamma_{h}} \mathbf{v}_{h}-\left(\mathbf{n}_{h} \times \mathbf{J}_{h}^{\mathrm{inc}}\right) \cdot \mathbf{v}_{h} d \Gamma_{h} .
$$

We set $\alpha_{j}=-A_{j} /\left(R_{0} B_{j}\right)$. The approximation space $V_{h}$ is the usual Nédélec's space of linear edge finite element [46]. If $N_{E}$ is the number of edges of $\Gamma_{h}$, then $\operatorname{dim}\left(V_{h}\right)=N_{E}$. The space $V_{h}^{p}$ is defined by: $V_{h}^{p}:=\times_{j=1}^{N_{p}} V_{h}$, with dimension $N_{E, p}:=N_{p} N_{E}$. The approximation space $Z_{h}$ is the nodal finite element space defined by

$$
Z_{h}=\left\{z_{h} \in \mathbf{H}^{-1 / 2}\left(\Gamma_{h}\right) \mid z_{h \mid T^{\ell}} \in \mathbb{P}_{1}\left(T^{\ell}\right), \forall \ell=1, \ldots, N_{T}\right\},
$$

where $\mathbb{P}_{1}$ is the space of linear functions. In particular, we have: $\operatorname{dim}\left(Z_{h}\right)=N_{V}$, where $N_{V}$ is the number of vertices of the triangulation. We also define: $Z_{h}^{p}:=\times_{j=1}^{N_{p}} Z_{h}$, with dimension $N_{V, p}:=$ $N_{p} N_{V}$. The discrete surface and damped wavenumber $k_{\varepsilon, h}$ is defined by: $k_{\varepsilon, h}:=k+\imath 0.39 k^{1 / 3} \mathcal{H}_{h}^{2 / 3}$, where $\mathcal{H}_{h}$ is a piecewise constant interpolation of the mean curvature $\mathcal{H}$ over $\Gamma_{h}$ on each triangle $T^{\ell}$ of the triangulation. In practice, the numerical computation of $\mathcal{H}_{h}$ is based on the finite element discretization of the following PDE

$$
2 \mathcal{H}=-\operatorname{div}_{\Gamma} \mathbf{n}, \text { on } \Gamma,
$$

Let us now introduce $\left(\mathbf{r}_{1}, \ldots, \mathbf{r}_{N_{E}}\right)$ (respectively $\left(\ell_{1}, \ldots, \ell_{N_{V}}\right)$ ) as the basis of $V_{h}$ (respectively of $\left.Z_{h}\right)$. Then, one gets for $\mathbf{v}_{h} \in V_{h}$ and $z_{h} \in Z_{h}$

$$
\mathbf{v}_{h}=\sum_{p=1}^{N_{E}} \mathbf{v}_{p} \mathbf{r}_{p} \quad \text { and } \quad z_{h}=\sum_{m=1}^{N_{V}} u_{m} \ell_{m} .
$$

Let us define the elementary integrals

$$
\left\{\begin{array}{l}
\mathbb{A}_{p q}=\int_{\Gamma_{h}} \mathbf{r}_{p} \cdot \mathbf{r}_{q} d \Gamma_{h}, \quad \mathbb{N}_{p q}=\int_{\Gamma_{h}} \frac{1}{k_{\varepsilon, h}^{2}} \operatorname{curl}_{\Gamma_{h}} \mathbf{r}_{p} \cdot \operatorname{curl}_{\Gamma} \mathbf{r}_{q} d \Gamma_{h}, \\
\mathbb{K}_{m n}=\int_{\Gamma_{h}} k_{\varepsilon, h}^{2} \ell_{m} \ell_{n} d \Gamma_{h}, \quad \mathbb{L}_{m q}=\int_{\Gamma_{h}} \nabla_{\Gamma_{h}} \ell_{m} \cdot \mathbf{r}_{q} d \Gamma_{h},
\end{array}\right.
$$

with $1 \leq p, q \leq N_{E}$ and $1 \leq m, n \leq N_{V}$. The associated matrices $\mathbb{A}$ and $\mathbb{N}$ are in $\mathcal{M}_{N_{E}, N_{E}}(\mathbb{C})$, $\mathbb{K} \in \mathcal{M}_{N_{V}, N_{V}}(\mathbb{C})$ and $\mathbb{L} \in \mathcal{M}_{N_{E}, N_{V}}(\mathbb{C})$. Under these notations, system (61) leads to solving the coupled linear system

$$
\mathbb{M}_{1} \mathbf{U}_{h}=\mathbf{F}_{1} \text {, }
$$

where we define

$$
\mathbb{M}_{1}=\left[\begin{array}{ccc}
\mathbb{A} & \mathbb{A}_{\boldsymbol{\alpha}} & \mathbf{0} \\
\mathbb{A}_{\boldsymbol{\alpha}}^{T} & \mathbb{A}_{\boldsymbol{\alpha}, \mathbf{B}} & \mathbb{A}_{\boldsymbol{\alpha}, \mathbf{B}}^{\mathbb{L}} \\
\mathbf{0} & \mathbb{A}_{\boldsymbol{\alpha}, \mathbf{B}}^{\mathbb{L}, T} & \mathbb{A}_{\boldsymbol{\alpha}, \mathbf{B}}^{\mathbb{K}}
\end{array}\right], \mathbf{U}_{h}=\left[\begin{array}{c}
\mathbf{M}_{h} \\
\boldsymbol{\Phi}_{h} \\
\boldsymbol{\rho}_{h}
\end{array}\right], \mathbf{F}_{1}=\left[\begin{array}{c}
\mathbf{F}_{1,1} \\
\mathbf{0} \\
\mathbf{0}
\end{array}\right]
$$

with

$$
\begin{aligned}
& \mathbb{A}_{\boldsymbol{\alpha}}:=\left(\left(\alpha_{j} \mathbb{A}\right)_{j=1, \ldots, N_{p}}\right) \in \mathcal{M}_{N_{E}, N_{E, p}}(\mathbb{C}), \\
& \mathbb{A}_{\boldsymbol{\alpha}, \mathbf{B}}:=\operatorname{diag}\left(\alpha_{j}\left(B_{j} \mathbb{N}-\mathbb{A}\right)_{j=1, \ldots, N_{p}}\right) \in \mathcal{M}_{N_{E, p}, N_{E, p}}(\mathbb{C}), \\
& \mathbb{A}_{\boldsymbol{\alpha}, \mathbf{B}}^{\mathbb{L}}:=\operatorname{diag}\left(\left(-\alpha_{j} B_{j} \mathbb{L}\right)_{j=1, \ldots, N_{p}}\right) \in \mathcal{M}_{N_{E, p}, N_{V, p}}(\mathbb{C}), \\
& \mathbb{A}_{\boldsymbol{\alpha}, \mathbf{B}}^{\mathbb{K}}:=\operatorname{diag}\left(\left(-\alpha_{j} B_{j} \mathbb{K}\right)_{j=1, \ldots, N_{p}}\right) \in \mathcal{M}_{N_{V, p}, N_{V, p}}(\mathbb{C}), \\
& \mathbf{F}_{1,1}:=-\frac{(\mathbb{N}-\mathbb{A})}{R_{0} Z_{0}}\left(\mathbf{n}_{h} \times \mathbf{J}_{h}^{\text {inc }}\right) \in \mathbb{C}^{N_{E}} .
\end{aligned}
$$


By $\mathbb{M}^{T}$, we designate the transposed matrix of $\mathbb{M}$. The sparse matrix $\mathbb{M}_{1}$ of size $n_{1, p} \times n_{1, p}$ (with $n_{1, p}:=N_{E}+N_{E, p}+N_{V, p}$ ) that defines the linear system (65) is non-definite positive, symmetrical and complex-valued. The vector fields $\mathbf{U}_{h}$ and $\mathbf{F}_{1}$ are in $\mathbb{C}^{n_{1, p}}$.

Concerning the second OSRC formulation (58), the globally unsymmetrical variational formulation is given by: find $\left(\mathbf{M}_{h}, \boldsymbol{\Phi}_{h}, \boldsymbol{\rho}_{h}\right) \in V_{h} \times V_{h}^{p} \times Z_{h}^{p}$ solution to

$$
\left\{\begin{aligned}
& \int_{\Gamma_{h}} \mathbf{M}_{h} \cdot \mathbf{v}_{h} d \Gamma_{h}+\sum_{j=1}^{N_{p}} \beta_{j} \int_{\Gamma_{h}} \phi_{h}^{j} \cdot \mathbf{v}_{h} d \Gamma_{h}=-\beta_{0} \int_{\Gamma_{h}} \mathbf{u}_{h} \cdot \mathbf{v}_{h} d \Gamma_{h} \\
& \int_{\Gamma_{h}}\left(q_{j} \phi_{h}^{j} \cdot \mathbf{w}_{h}^{j}+\frac{1}{k_{\varepsilon, h}^{2}} \operatorname{curl}_{\Gamma_{h}} \phi_{h}^{j} \cdot \operatorname{curl}_{\Gamma_{h}} \mathbf{w}_{h}^{j}\right) d \Gamma_{h}-\int_{\Gamma_{h}} \nabla_{\Gamma_{h}} \rho_{h}^{j} \cdot \mathbf{w}_{h}^{j} d \Gamma_{h}=-\int_{\Gamma_{h}} \mathbf{u}_{h} \cdot \mathbf{w}_{h}^{j} d \Gamma_{h}, \quad j=1, \ldots, N_{p} \\
& \int_{\Gamma_{h}}\left(k_{\varepsilon, h}^{2} \rho_{h}^{j} z_{h}^{j}+\phi_{h}^{j} \cdot \nabla_{\Gamma_{h}} z_{h}^{j}\right) d \Gamma_{h}=0, \quad j=1, \ldots, N_{p}
\end{aligned}\right.
$$

where $\mathbf{v}_{h}$ and $\mathbf{w}_{h}^{j}$ are in $V_{h}, z_{h}^{j} \in Z_{h}, \beta_{j}=r_{j} / Z_{0}$, for $j=1, \ldots, N_{p}$. We recall that the entry $\mathbf{u}_{h}$ is given by the equation (62). There is a major difference between systems (61) and (68). Indeed, system (61) fully couples all the components of $\left(\mathbf{M}_{h}, \boldsymbol{\Phi}_{h}, \boldsymbol{\rho}_{h}\right)$ while, for 68 , only $\boldsymbol{\phi}_{h}^{j}$ and $\rho_{h}^{j}$ are related by the two last equations of (68), for a fixed $j=1, \ldots, N_{p}$. More precisely, we first have to solve (possibly in parallel) $N_{p}$ decoupled symmetrical complex-valued sparse linear systems

$$
\mathbb{M}_{2}^{j}\left[\begin{array}{c}
\phi_{h}^{j} \\
\rho_{h}^{j}
\end{array}\right]:=\left[\begin{array}{cc}
\mathbb{B}_{q_{j}} & \mathbb{L} \\
\mathbb{L}^{T} & \mathbb{K}
\end{array}\right]\left[\begin{array}{c}
\phi_{h}^{j} \\
\rho_{h}^{j}
\end{array}\right]=\left[\begin{array}{c}
\mathbf{F}_{2,1} \\
0
\end{array}\right], j=1, \ldots, N_{p},
$$

with $\mathbb{B}_{q_{j}}:=-\left(q_{j} \mathbb{A}+\mathbb{N}\right)$ and $\mathbf{F}_{2,1}=(\mathbb{N}-\mathbb{A})\left(\mathbf{n}_{h} \times \mathbf{J}_{h}^{\text {inc }}\right)$. The square matrix $\mathbb{M}_{2}^{j}$ has a size $n_{2} \times n_{2}$, with $n_{2}:=N_{E}+N_{V}$, which is much less than for $\mathbb{M}_{1}$ and $N_{p}$-independent. Then, in a second step, we obtain the unknown discrete magnetic surface current $\mathbf{M}_{h}$ by solving the linear system

$$
\mathbb{A} \mathbf{M}_{h}=\frac{r_{0}}{Z_{0}}(\mathbb{A}-\mathbb{N})\left(\mathbf{n}_{h} \times \mathbf{J}_{h}^{\mathrm{inc}}\right)-\mathbb{A}\left(\sum_{j=1}^{N_{p}} \beta_{j} \phi_{h}^{j}\right),
$$

of size $N_{E} \times N_{E}$ which is again much less than for $\mathbb{M}_{1}$. As a consequence, we can expect that this second approach, based on (68), outperforms (61) in terms of computational times.

\section{Numerical results}

We now consider various scattering problems by PEC and impedance scatterers to numerically validate the MtE operators in the context of OSRCs methods. The objects are illuminated by an electromagnetic plane wave defined by

$$
\mathbf{E}^{\text {inc }}(\mathbf{x})=\mathbf{p} e^{\imath \mathbf{d} \cdot \mathbf{x}},
$$

where $\mathbf{p}$ is the polarization vector and $\mathbf{d}=\left(\sin \left(\theta^{\text {inc }}\right) \cos \left(\phi^{\text {inc }}\right), \sin \left(\theta^{\text {inc }}\right) \sin \left(\phi^{\text {inc }}\right), \cos \left(\theta^{\text {inc }}\right)\right)^{t}$ is the incidence vector such that $\mathbf{p} \cdot \mathbf{d}=0$. To evaluate the method, we consider the bistatic Radar Cross Section (RCS) given by

$$
\operatorname{RCS}(\theta, \phi)=10 \log _{10}\left(\left|\mathbf{E}_{\infty}(\hat{\mathbf{x}}(\theta, \phi))\right|^{2}\right)(\mathrm{dB}),
$$

where $\mathbf{E}_{\infty}$ is defined by relation (6) and the point $\hat{\mathbf{x}}$ is given on the unit sphere $\mathbb{S}_{1}$ by its spherical coordinates: $\hat{\mathbf{x}}(\theta, \phi)=(\sin (\theta) \cos (\phi), \sin (\theta) \sin (\phi), \cos (\theta))^{T}$ (the angles are given in degrees here). 
Before assessing the accuracy of the OSRC methods, let us take a look at the convergence of the finite element approximations and their computational performance. We recall that we have two formulations. The first one, based on system (66)-(67), essentially uses the square-root approximation (50) of the OSRC operators. For this reason, this formulation is called Sqrt in the sequel. The second formulation uses the approximation (57) and leads to the decoupled systems (69)-(70) of surface PDEs. The resulting method is called Isqrt. Concerning the finite element methods, we introduce the density of discretization points per wavelength $n_{\lambda}=\lambda / h$. We consider the scattering problem of an incident plane wave with incidence angle $\left(\theta^{\text {inc }}, \phi^{\text {inc }}\right)=(180,180)$ by the unit sphere $\mathbb{S}_{1}$. We report in Table 2 the error $\left\|\widetilde{\mathbf{M}}-\mathbf{M}_{h}\right\|_{L^{2}\left(\mathbb{S}_{1}\right)} /\|\widetilde{\mathbf{M}}\|_{L^{2}\left(\mathbb{S}_{1}\right)}$ (in \%) between the analytical OSRC surface field $\widetilde{\mathbf{M}}$ given by 29 and the numerical field $\mathbf{M}_{h}$ provided by 70 , with respect to the wavenumber $k$ and number $N_{p}$ of Padé functions. The discretization density is fixed to $n_{\lambda}=21$. We can see that the finite element approximation is very accurate in all the situations (for $N_{p} \geq 2$ ). Furthermore, the accuracy of the OSRC method is quite optimal for $N_{p}=2$ auxiliary functions whatever is the wavenumber $k$. A similar accuracy is observed for Sqrt. Other simulations show that $n_{\lambda}=10$ already leads to correct results and that finer meshes improve the accuracy.

\begin{tabular}{|c||c|c|c|c|c|}
\hline$k$ & $N_{p}=0$ & $N_{p}=2$ & $N_{p}=4$ & $N_{p}=8$ & $N_{p}=12$ \\
\hline \hline$\pi$ & 29.18 & 0.553 & 0.521 & 0.514 & 0.513 \\
\hline $2 \pi$ & 37.68 & 0.426 & 0.395 & 0.393 & 0.393 \\
\hline $4 \pi$ & 43.93 & 0.408 & 0.340 & 0.340 & 0.340 \\
\hline $6 \pi$ & 46.57 & 0.450 & 0.332 & 0.331 & 0.331 \\
\hline $8 \pi$ & 48.11 & 0.510 & 0.330 & 0.329 & 0.329 \\
\hline
\end{tabular}

Table 2: Accuracy of the finite element method for Isqrt in the case of the unit sphere $\mathbb{S}_{1}$ vs. $k$ and $N_{p}$. The discretization density is $n_{\lambda}=21$.

Let us now consider the performances of the different OSRC formulations. We report in Table 3 the total CPU time (in seconds) for the two formulations Sqrt and Isqrt. All the tests run on an Intel (R) Xeon at 2.27Ghz (8Go memory). The linear systems are solved by the sparse direct solver MUMPS*. In the case of formulation Sqrt, the linear system to solve, i.e. system (65), couples the $N_{p}$ auxiliary functions. We have seen that this system can be quite large most particularly if $N_{p} \gg 1$ since then $n_{1, p}$ is large too. In the case of formulation Isqrt, the $N_{p}$ systems defined by $\mathbb{M}_{2}^{j}, j=1, \ldots, N_{p}$, are decoupled (see Eq. 69) ) and of fixed size $n_{2} \times n_{2}$ with respect to $N_{p}$. From these numerical results, we clearly see that Isqrt requires less memory and is much faster than Sqrt for an equivalent accuracy. As a consequence, the Isqrt OSRC formulation outperforms the Sqrt OSRC formulation. For this reason, we only use the Isqrt formulation in the sequel of the paper.

Let us now come to the evaluation of the OSRC methods compared to a reference solution for various scatterers, frequencies and incidence angles. First, the reference solution consists in the full Maxwell's equations solution obtained through a three-dimensional finite element method with fine mesh. The solution to the large size associated linear system is computed by using a Domain Decomposition Method when the use of a direct sparse solver is limited. For comparison, we essentially report the bistatic RCS (dB) and the (real part of) surface current $\mathbf{M}_{h}$.

In the first example, we consider on Figure 4 the bistatic RCS for the scattering problem of an incident electromagnetic plane wave by the PEC sphere $\mathbb{S}_{1}$ for $k=\pi$ and $k=8 \pi$. In both cases, we observe that the OSRC solution (with $N_{p}=2$ ) is a very good approximation of the reference solution obtained for a cheap computational cost. In the illuminated forward scattering directions, the accuracy of the OSRC solution is satisfactory. The discrepancies are mainly observable in the

*http://graal.ens-lyon.fr/MUMPS/ 


\begin{tabular}{|c|c|c|c|c|c|}
\hline & & $N_{p}=1$ & $N_{p}=2$ & $N_{p}=4$ & $N_{p}=8$ \\
\hline \multicolumn{6}{|c|}{$k=6 \pi, n_{\lambda}=11$} \\
\hline \multirow[t]{2}{*}{ Sqrt } & $\overline{n_{1, p}}$ & 108383 & 170317 & 294185 & 5451921 \\
\hline & CPU (sec.) & 18 & 29 & 84 & 416 \\
\hline \multirow[t]{2}{*}{ Isqrt } & $n_{2}$ & 61934 & 61934 & 61934 & 61934 \\
\hline & CPU (sec.) & 19 & 28 & 44 & 77 \\
\hline \multicolumn{6}{|c|}{$k=6 \pi, n_{\lambda}=21$} \\
\hline \multirow[t]{2}{*}{ Sqrt } & $n_{1, p}$ & 404056 & 634946 & 1096726 & 2020286 \\
\hline & CPU (sec.) & 84 & 146 & 515 & 2778 \\
\hline \multirow[t]{2}{*}{ Isqrt } & $n_{2}$ & 230890 & 230890 & 230890 & 230890 \\
\hline & CPU (sec.) & 90 & 124 & 194 & 333 \\
\hline \multicolumn{6}{|c|}{$k=8 \pi, n_{\lambda}=11$} \\
\hline \multirow[t]{2}{*}{ Sqrt } & 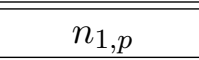 & 197388 & 310182 & 535770 & 9986946 \\
\hline & CPU (sec.) & 35 & 60 & 184 & 928 \\
\hline \multirow[t]{2}{*}{ Isqrt } & $n_{2}$ & 112794 & 112794 & 112794 & 112794 \\
\hline & CPU (sec.) & 37 & 53 & 85 & 149 \\
\hline
\end{tabular}

Table 3: Size of the linear systems and CPU time of the Sqrt and Isqrt OSRC methods with respect to the different parameters $k, n_{\lambda}$ and $N_{p}$ (for the scattering by the unit PEC sphere).

deep shadow zone. This is due to the fact that the complex phase of the grazing waves is not completely correctly modeled in the OSRC operators. Indeed, the $\varepsilon$ parameter defined by relation 43 is used to increase the accuracy in the shadow zone but in an approximate way. Finally, the analytical (i.e. Mie series) and numerical (i.e. finite element for $n_{\lambda}=25$ ) OSRC solutions superpose which means that the full numerical OSRC method is accurate. Increasing $N_{p}$ does not improve the accuracy here, which means that $N_{p}=2$ is optimal for the OSRC method.
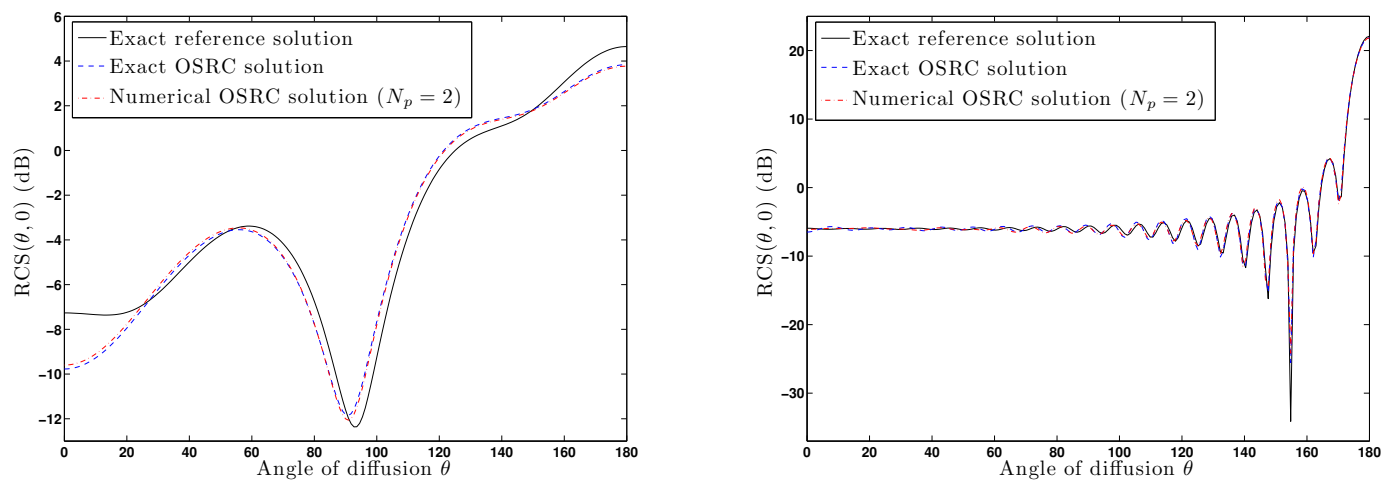

Figure 4: Bistatic RCS for the PEC unit sphere $\mathbb{S}_{1}$ illuminated by an incident electromagnetic plane wave with $\left(\theta^{\text {inc }}, \phi^{\text {inc }}\right):=(180,180)$ at $k=\pi$ (right) and $k=8 \pi$ (left).

The second test case consists in the scattering problem by an ellipsoid with semi-axes $a=1$, $b=0.5$ and $c=b$ along the $x$-, $y$ - and $z$-directions, respectively. We report on Figures 5 and 6 the real parts of the reference and (numerical) OSRC (for $n_{\lambda}=30$ ) surface currents $\mathbf{M}$ for two couples of incidence angles and $k=3 \pi$. We remark that globally the surface fields are correctly computed even if some errors are again observed in the shadow zone. When computing the corresponding bistatic RCS (see Figure 7), we see that we obtain a correct approximation for all angles, with an optimal accuracy for $N_{p}=2$. 

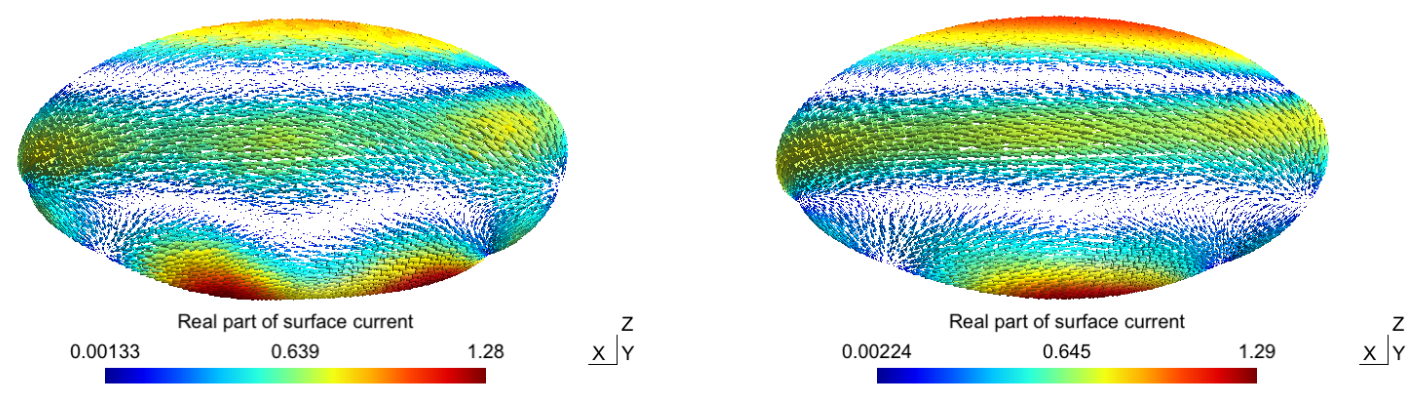

Figure 5: Scattering of a plane wave by a PEC prolate ellipsoid; real part of the surface current $\mathbf{M}$ for $k=3 \pi$ and $\left(\theta^{\text {inc }} \phi^{\text {inc }}\right)=(180,180)$ deg.: reference solution (left) and numerical OSRC solution for $N_{p}=2$ (right picture).
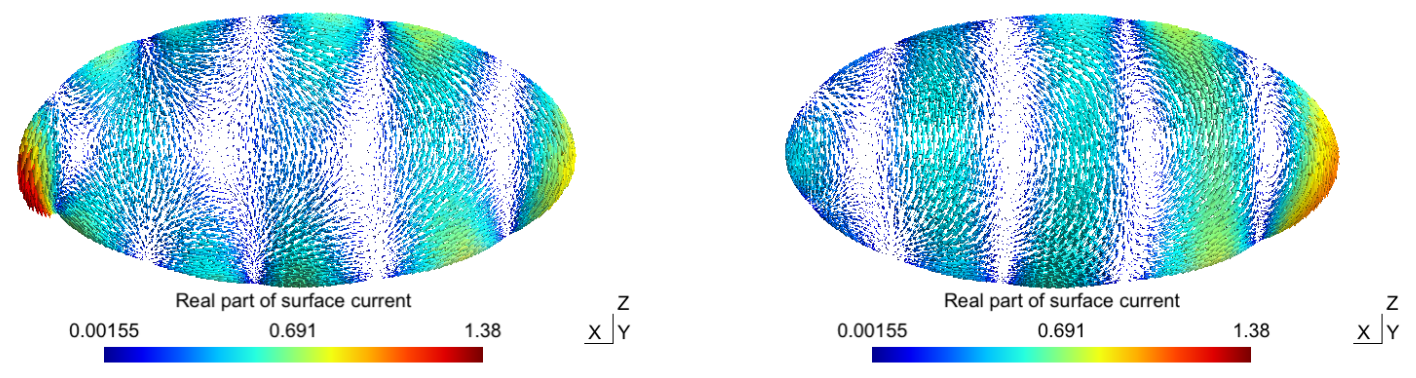

Figure 6: Scattering of a plane wave by a PEC prolate ellipsoid; real part of the surface current $\mathbf{M}$ for $k=3 \pi$ and $\left(\theta^{\text {inc }} \phi^{\text {inc }}\right)=(90,0)$ deg.: reference solution (left) and numerical OSRC solution for $N_{p}=2$ (right).

The third obstacle is a semi concave sphere (see Figure 8). We report on figures 9 the RCS for $k=3 \pi$ in two situations. The first example (Figure 9, left) consists in considering $\left(\theta^{\text {inc }}, \phi^{\text {inc }}\right)=$ $(180,180)$ degrees. First, we see that the RCS is correct in the forward scattering direction and that errors occur in the shadow zone. Furthermore, the accuracy is better in the convex transition zone than in the concave part. The second example (Figure 9, right) takes $\left(\theta^{\text {inc }}, \phi^{\text {inc }}\right)=(90,0)$. We observe that the main errors arise in the non convex part $(\theta=90$ degrees $)$ while scattering in the convex part $(\theta=270$ degrees) is very well reproduced.

In the next example, we consider the scattering problem by a PEC cube of side one, centered at the origin. We report on figure 10 the bistatic RCS for $k=2 \pi$ and $\left(\theta^{\text {inc }}, \phi^{\text {inc }}\right)=(180,180)$. We remark that the RCS is correctly computed. For comparison, we also give the RCS computed by using a global constant mean curvature $\mathcal{H}^{e q v}=\sqrt{2}$ in relation $(43)$ and the numerical one. We see that considering the numerical curvature improves the precision of the method for some angles.

Finally, we consider the scattering problem by a scatterer with an impedance boundary condition. Therefore, we have a boundary condition of the following form [55]

$$
\mathbf{n} \times \mathbf{J}+\mathcal{Z}\left(Z_{0} \mathbf{M}\right)=\mathbf{g} \text {, on } \Gamma,
$$

where $\mathcal{Z}$ is a given surface operator. Here, we restrict the study to the case where $\mathcal{Z}$ is a given complex valued constant but more general operators can be considered, our approach being direct to extend. Using the Isqrt OSRC approach, we have to solve for $\mathbf{g}=-\mathbf{n} \times \mathbf{J}^{\text {inc }}-\mathcal{Z} Z_{0} \mathbf{M}^{\text {inc }}$, the equation

$$
\left(\boldsymbol{\Lambda}_{1, \varepsilon}-\mathcal{Z} Z_{0} \boldsymbol{\Lambda}_{2, \varepsilon}\right) \mathbf{M}=-\boldsymbol{\Lambda}_{2, \varepsilon} \mathbf{g}
$$

Unlike the PEC case, the problem is fully coupled in terms of surface fields $\mathbf{M}$ and $\mathbf{J}$. Whatever is the OSRC formulation, i.e. Isqrt or Sqrt, introducing auxiliary functions requires the solution to 

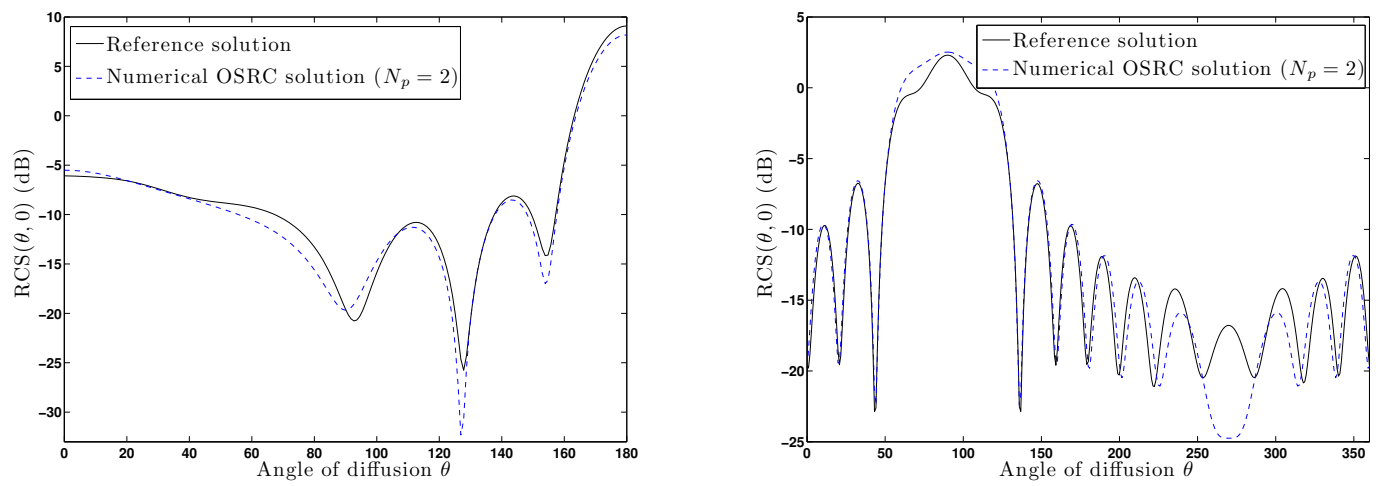

Figure 7: Bistatic RCS for the PEC prolate ellipsoid illuminated by an incident electromagnetic plane wave with $k=3 \pi$ : $\left(\theta^{\text {inc }}, \phi^{\text {inc }}\right)=(180,180)$ deg. $($ left $)$ and $\left(\theta^{\text {inc }}, \phi^{\text {inc }}\right)=(90,0)$ deg. (right).

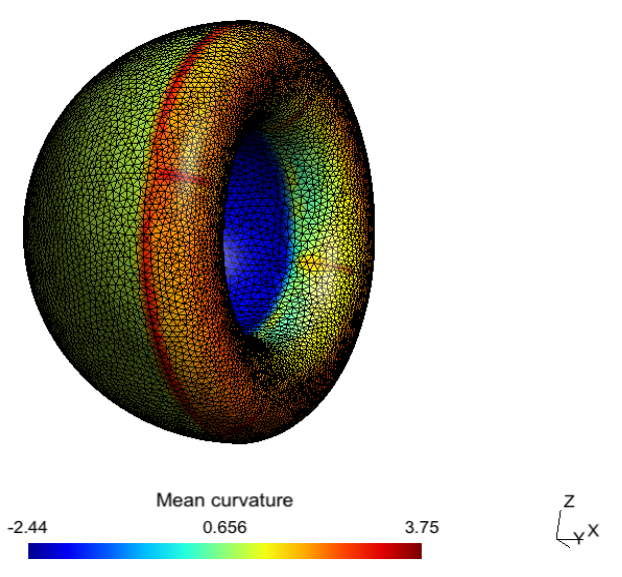

Figure 8: The semi concave sphere.

a coupled system of PDEs over $\Gamma$ similarly to Sqrt. Decoupling the fields could be possible by using a numerical iterative procedure that we do not study here. To show one example of computation, we fix $\Gamma=\mathbb{S}_{1}$ for an incident plane wave $\mathbf{E}^{\text {inc }}(\mathbf{x})=(1,0,0)^{t} e^{-i k x_{3}}$. This allows us to obtain an analytical solution for the exact solution. For the OSRC solution, we use the full numerical approximation by adapting the previous finite element formulations. We choose $\mathcal{Z}:=1+0.5 \imath$. We report on Figure 11 the RCS for $k=4 \pi$. We observe again a very good agreement between the exact and approximate solutions even is small oscillations occur in the shadow zone. Figure 12 gives (the real part of) the surface fields $\mathbf{M}$ for the reference and approximate methods. We see that the fields are correctly reproduced even if small errors occur in the shadow zone.

\section{Conclusion}

The aim of this paper was to propose some new local approximate accurate magnetic-to-electric surface operators for the three-dimensional time harmonic Maxwell's equations. These operators are built by using a diagonalization procedure, a regularization technique and a local operator representation based on rational complex Padé approximants. These operators are next numerically approximated by using finite element methods. The resulting techniques, applied in the context of OSRC methods, provide fast accurate surface fields and associated RCS for various obstacles.

One of the main application of these new operators is to consider them as tools for improving 

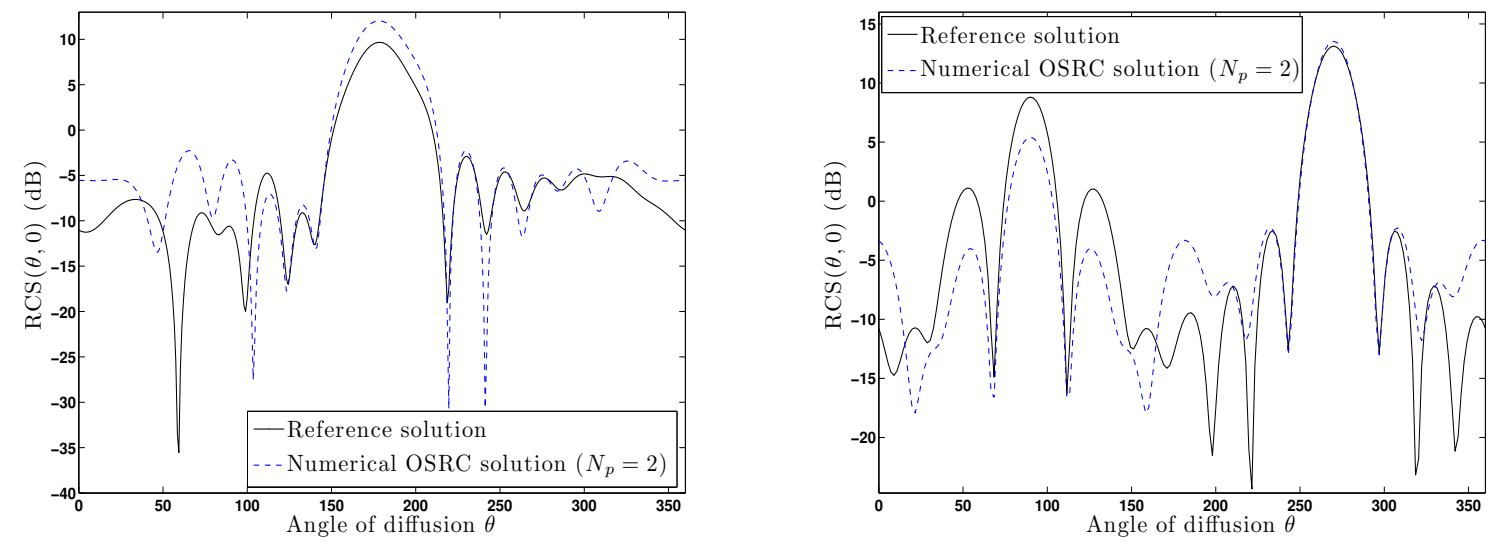

Figure 9: Bistatic RCS for the PEC semi concave sphere illuminated by an incident electromagnetic plane wave with $k=3 \pi$ : $\left(\theta^{\text {inc }}, \phi^{\text {inc }}\right)=(180,180)$ deg. $($ left $)$ and $\left(\theta^{\text {inc }}, \phi^{\text {inc }}\right)=(90,180)$ deg. (right).

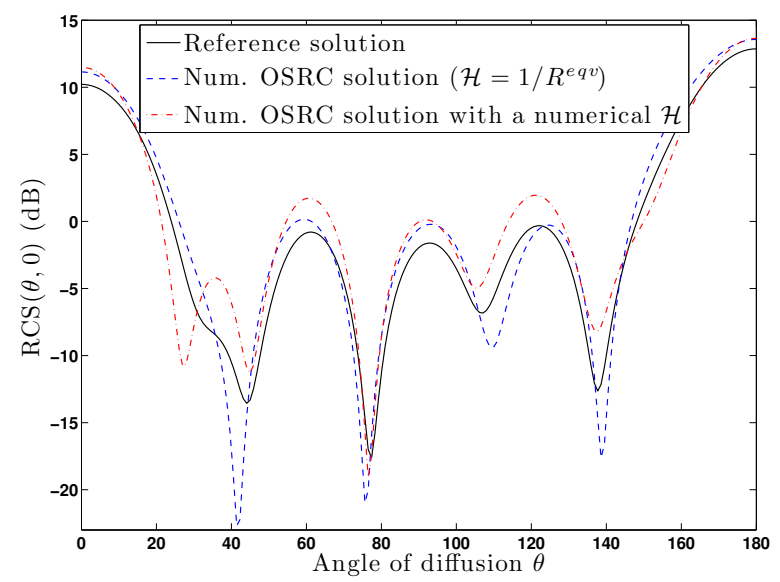

Figure 10: Bistatic RCS for the PEC unit cube illuminated by an incident electromagnetic plane wave with $k=2 \pi$ and $\left(\theta^{\text {inc }}, \phi^{\text {inc }}\right)=(180,180)$ degrees.

other numerical methods. Let us e.g. mention preconditioning techniques and well conditioned integral equation formulations [11, 12, 13, 10, 9, 21, 22, 2, 19, 39] or robust domain decomposition methods [33, 30, 31, 42, 41, 50, 49, 20]. They can also be applied as Absorbing Boundary Conditions (ABCs) [7, 37, 52, 16, 15, 44, 57, 17, 56, 35, 34] on a general convex shape surrounding the scatterer. Some of these aspects will be the subject of forthcoming studies.

\section{Acknowledgements.}

This work was supported in part by the French ANR grant MicroWave NT09_460489 ("Programme Blanc" call) and the "EADS Foundation" (High-BRID project, grant reference 089-10091006).

\section{References}

[1] M. Abramowitz and I. Stegun. Handbook of Mathematical Functions: With Formulas, Graphs, and Mathematical Tables. Applied mathematics series. Dover Publications, 1964. 


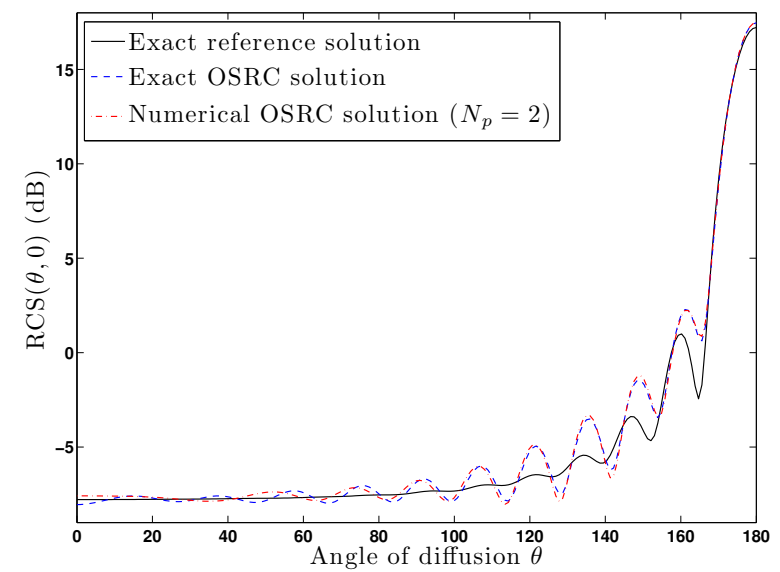

Figure 11: Bistatic RCS for the impedance unit sphere illuminated by an incident electromagnetic plane wave with $k=4 \pi$.
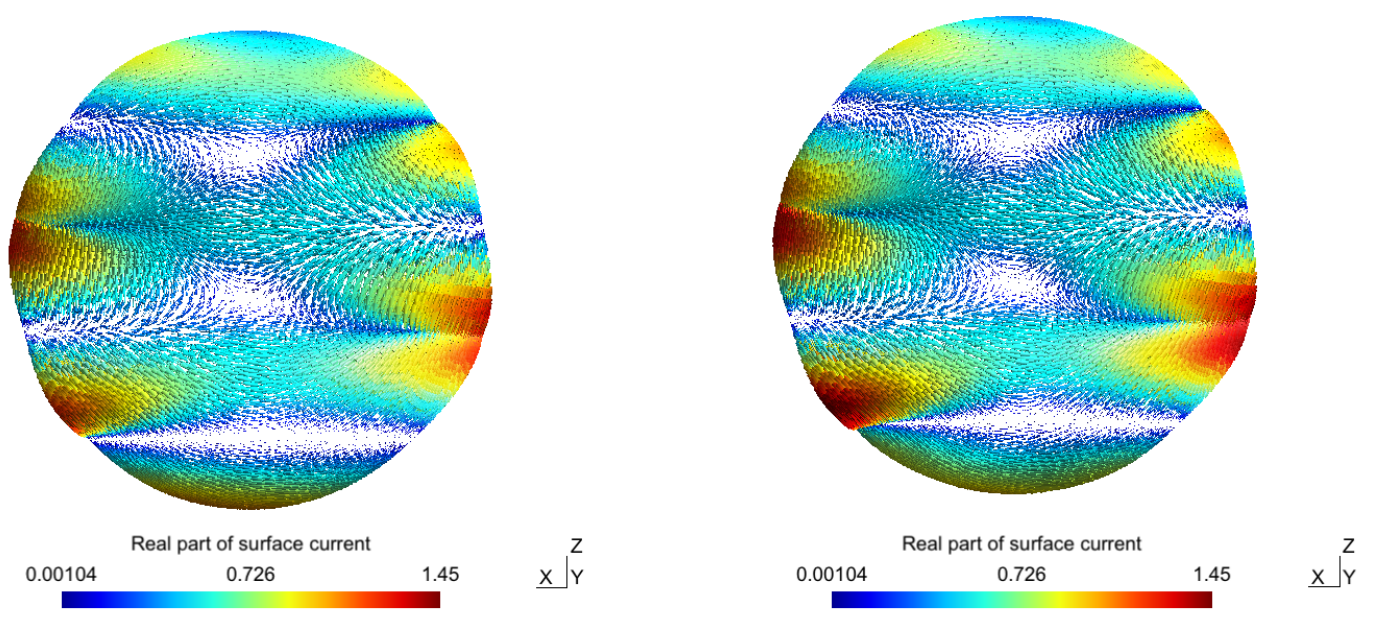

Figure 12: Scattering of a plane wave by an impedance sphere; real part of the surface current $\mathbf{M}$ for $k=4 \pi$ : reference solution (left) and numerical OSRC solution for $N_{p}=2$ (right).

[2] F. Alouges, S. Borel, and D. P. Levadoux. A stable well-conditioned integral equation for electromagnetism scattering. J. Comput. Appl. Math., 204(2):440-451, 2007.

[3] H. Ammari. Scattering of waves by thin periodic layers at high frequencies using the on-surface radiation condition method. IMA J. Appl. Math., 60:199-215, 1997.

[4] I. Andronov, D. Bouche, and F. Molinet. Asymptotic and hybrid methods in electromagnetics, volume 48 of IEE Electromagnetic Waves Series. Institution of Electrical Engineers (IEE), London, 2005.

[5] X. Antoine. Fast approximate computation of a time-harmonic scattered field using the onsurface radiation condition method. IMA J. Appl. Math., 66(1):83-110, 2001.

[6] X. Antoine. Advances in the on-surface radiation condition method: Theory, numerics and applications. In Computational Methods for Acoustics Problems, pages 169-194. Saxe-Coburg Publications, 2008. 
[7] X. Antoine, H. Barucq, and A. Bendali. Bayliss-Turkel-like radiation conditions on surfaces of arbitrary shape. J. Math. Anal. Appl., 229(1):184-211, 1999.

[8] X. Antoine, H. Barucq, and L. Vernhet. High-frequency asymptotic analysis of a dissipative transmission problem resulting in generalized impedance boundary conditions. Asymptot. Anal., 26(3-4):257-283, 2001.

[9] X. Antoine, A. Bendali, and M. Darbas. Analytic preconditioners for the electric field integral equation. Internat. J. Numer. Methods Engrg., 61(8):1310-1331, 2004.

[10] X. Antoine, A. Bendali, and M. Darbas. Analytic preconditioners for the boundary integral solution of the scattering of acoustic waves by open surfaces. J. Comput. Acoust., 13(3):477498, 2005.

[11] X. Antoine and M. Darbas. Alternative integral equations for the iterative solution of acoustic scattering problems. Quarterly Journal of Mechanics and Applied Mathematics, 1(58):107-128, 2005 .

[12] X. Antoine and M. Darbas. Generalized Combined Field Integral Equations for the iterative solution of the three-dimensional Helmholtz equation. Mathematical Modelling and Numerical Analysis, 1(41):147-167, 2007.

[13] X. Antoine and M. Darbas. Integral Equations and Iterative Schemes for Acoustic Scattering Problems. Saxe-Coburg Editors, to appear, 2014.

[14] X. Antoine, M. Darbas, and Y. Y. Lu. An improved surface radiation condition for highfrequency acoustic scattering problems. Comput. Methods Appl. Mech. Engrg., 195(3336):4060-4074, 2006.

[15] H. Barucq, R. Djellouli, and A. Saint-Guirons. High-frequency analysis of the efficiency of a local approximate DtN2 boundary condition for prolate spheroidal-shaped boundaries. Wave Motion, 47(8):583-600, 2010.

[16] H. Barucq, R. Djellouli, and A. Saint-Guirons. Three-dimensional approximate local DtN boundary conditions for prolate spheroid boundaries. J. Comput. Appl. Math., 234(6):1810$1816,2010$.

[17] A. Bayliss and E. Turkel. Radiation boundary conditions for wave-like equations. Comm. Pure Appl. Math., 33(6):707-725, 1980.

[18] J.-P. Bérenger. A perfectly matched layer for the absorption of electromagnetic waves. $J$. Comput. Phys., 114(2):185-200, 1994.

[19] S. Borel, D. P. Levadoux, and F. Alouges. A new well-conditioned integral formulation for Maxwell equations in three dimensions. IEEE Trans. Antennas and Propagation, 53(9):29953004, 2005.

[20] Y. Boubendir, X. Antoine, and C. Geuzaine. A quasi-optimal non-overlapping domain decomposition algorithm for the Helmholtz equation. Journal of Computational Physics, 2(231):262$280,2012$.

[21] O. Bruno, T. Elling, R. Paffenroth, and C. Turc. Electromagnetic integral equations requiring small numbers of Krylov-subspace iterations. J. Comput. Phys., 228(17):6169-6183, 2009. 
[22] O. Bruno, T. Elling, and C. Turc. Regularized integral equations and fast high-order solvers for sound-hard acoustic scattering problems. Internat. J. Numer. Methods Engrg., 91(10):10451072, 2012.

[23] S. N. Chandler-Wilde, I. G. Graham, S. Langdon, and E. A. Spence. Numerical-asymptotic boundary integral methods in high-frequency acoustic scattering. Acta Numer., 21:89-305, 2012 .

[24] W. Chew, J.-M. Jin, E. Michielssen, and J. Song. Fast and Efficient Algorithms in Computational Electromagnetics. Artech House Antennas and Propagation Library, Norwood, 2001.

[25] W. Chew and W. Weedon. A 3D Perfectly matched medium from modified Maxwell's equations with stretched coordinates. Microwave and Optical Technology Letters, 7(13):599-604, SEP 1994.

[26] D. Colton and R. Kress. Integral equation methods in scattering theory. Pure and applied mathematics. Wiley, 1993.

[27] M. Darbas. Generalized combined field integral equations for the iterative solution of the three-dimensional Maxwell equations. Appl. Math. Lett., 19(8):834-839, 2006.

[28] M. Darbas, E. Darrigrand, and Y. Lafranche. Combining analytic preconditioner and fast multipole method for the 3-D Helmholtz equation. J. Comput. Phys., 236:289-316, 2013.

[29] E. Darve. The fast multipole method: numerical implementation. J. Comput. Phys., 160(1):195-240, 2000.

[30] V. Dolean, M. J. Gander, and L. Gerardo-Giorda. Optimized Schwarz methods for Maxwell's equations. SIAM J. Sci. Comput., 31(3):2193-2213, 2009.

[31] M. El Bouajaji, V. Dolean, M. J. Gander, and S. Lanteri. Optimized Schwarz methods for the time-harmonic Maxwell equations with damping. SIAM J. Sci. Comput., 34(4):A2048-A2071, 2012 .

[32] B. Engquist and A. Majda. Radiation boundary conditions for acoustic and elastic wave calculations. Comm. Pure Appl. Math., 32(3):314-358, 1979.

[33] M. J. Gander, F. Magoulès, and F. Nataf. Optimized Schwarz methods without overlap for the Helmholtz equation. SIAM J. Sci. Comput., 24(1):38-60 (electronic), 2002.

[34] D. Givoli. High-order local non-reflecting boundary conditions: a review. Wave Motion, 39(4):319-326, APR 2004.

[35] T. Hagstrom. New results on absorbing layers and radiation boundary conditions. In Ainsworth, M and Davies, P and Duncan, D and Martin, P and Rynne, B, editor, Topics in Computational Wave Propagation: Direct and Inverse Problems, volume 31 of Lecture Notes in Computational Science and Engineering, pages 1-42, 2003. Symposium on Computational Methods for Wave Propagation in Direct Scattering, Univ Durham, Durham, England, Jul $15-25,2002$.

[36] J. Jin. The Finite Element Method in Electromagnetics. John Wiley \& Sons, Incorporated, New York, second edition, 2002.

[37] R. Kechroud, X. Antoine, and A. Soulaïmani. Numerical accuracy of a Padé-type non-reflecting boundary condition for the finite element solution of acoustic scattering problems at highfrequency. Internat. J. Numer. Methods Engrg., 64(10):1275-1302, 2005. 
[38] G. A. Kriegsmann, A. Taflove, and K. R. Umashankar. A new formulation of electromagnetic wave scattering using an on-surface radiation boundary condition approach. IEEE Trans. Antennas and Propagation, 35(2):153-161, 1987.

[39] D. P. Levadoux and B. L. Michielsen. Nouvelles formulations intégrales pour les problèmes de diffraction d'ondes. M2AN Math. Model. Numer. Anal., 38(1):157-175, 2004.

[40] Y. Liu. Fast Multipole Boundary Element Method. Theory and Applications in Engineering. Cambridge University Press, 2009.

[41] F. Magoulès, P. Iványi, and B. H. V. Topping. Non-overlapping Schwarz methods with optimized transmission conditions for the Helmholtz equation. Comput. Methods Appl. Mech. Engrg., 193(45-47):4797-4818, 2004.

[42] F. Magoulès and F.-X. Roux. Dirichlet-to-Neumann maps for domain decomposition methods: a unified approach. In Domain decomposition methods: theory and applications, volume 25 of GAKUTO Internat. Ser. Math. Sci. Appl., pages 123-145. Gakkōtosho, Tokyo, 2006.

[43] A. Majda and S. Osher. Reflection of singularities at the boundary. Comm. Pure Appl. Math., 28(4):479-499, 1975.

[44] M. Medvinsky and E. Turkel. On surface radiation conditions for an ellipse. J. Comput. Appl. Math., 234(6):1647-1655, 2010.

[45] F. Milinazzo, C. Zala, and G. Brooke. Rational square-root approximations for parabolic equation algorithms. Journal of the Acoustical Society of America, 101(2):760-766, FEB 1997.

[46] P. Monk. Finite Element Methods for Maxwell's Equations. Numerical Mathematics and Scientific Computation. Oxford University, 2003.

[47] R. Murch. The on-surface radiation condition applied to three-dimensional convex objects. IEEE Trans. Antennas and Propagation, 41(5):651-658, 1993.

[48] J.-C. Nédélec. Acoustic and electromagnetic equations, volume 144 of Applied Mathematical Sciences. Springer-Verlag, New York, 2001. Integral representations for harmonic problems.

[49] Z. Peng and J.-F. Lee. A scalable nonoverlapping and nonconformal domain decomposition method for solving time-harmonic Maxwell equations in $\mathbb{R}^{3}$. SIAM J. Sci. Comput., 34(3):A1266-A1295, 2012.

[50] Z. Peng, V. Rawat, and J.-F. Lee. One way domain decomposition method with second order transmission conditions for solving electromagnetic wave problems. J. Comput. Phys., 229(4):1181-1197, 2010.

[51] S. Pernet. A well-conditioned integral equation for iterative solution of scattering problems with a variable Leontovitch boundary condition. M2AN Math. Model. Numer. Anal., 44(4):781-801, 2010.

[52] R. C. Reiner, Jr., R. Djellouli, and I. Harari. The performance of local absorbing boundary conditions for acoustic scattering from elliptical shapes. Comput. Methods Appl. Mech. Engrg., 195(29-32):3622-3665, 2006.

[53] R. Roxburgh. Electromagnetic scattering from a right-circular cylinder using a surface radiation condition. IMA J. Appl. Math., 59:221-230, 1997. 
[54] Y. Saad. Iterative methods for sparse linear systems. Society for Industrial and Applied Mathematics, Philadelphia, PA, second edition, 2003.

[55] T. B. Senior and J. L. Volakis. Approximate Boundary Conditions in Electromagnetics. IET, London, 1995.

[56] S. Tsynkov. Numerical solution of problems on unbounded domains. A review. Applied Numerical Mathematics, 27(4):465-532, AUG 1998.

[57] E. Turkel. Boundary conditions and iterative schemes for the Helmholtz equation in unbounded region. In Computational Methods for Acoustics Problems, pages 127-158. Saxe-Coburg Publications, 2008. 